\title{
THE
}

$11-2014$

\section{On the Generation of Roll Vortices Due to the Inflection Point Instability of the Hurricane Boundary Layer Flow}

Kun Gao

Isaac Ginis

University of Rhode Island, iginis@uri.edu

Follow this and additional works at: https://digitalcommons.uri.edu/gsofacpubs

Terms of Use

All rights reserved under copyright.

\section{Citation/Publisher Attribution}

Gao, Kun; Ginis, Isaac. 2014. "On the Generation of Roll Vortices due to the Inflection Point Instability of the Hurricane Boundary Layer Flow." Journal of the Atmospheric Sciences. 71(11): 4292-4307.

Available at: http://dx.doi.org/10.1175/JAS-D-13-0362.1

This Article is brought to you for free and open access by the Graduate School of Oceanography at DigitalCommons@URI. It has been accepted for inclusion in Graduate School of Oceanography Faculty Publications by an authorized administrator of DigitalCommons@URI. For more information, please contact digitalcommons-group@uri.edu. 


\title{
¿ On the Generation of Roll Vortices due to the Inflection Point Instability of the Hurricane Boundary Layer Flow
}

\author{
KUN GAO AND ISAAC GINIS \\ Graduate School of Oceanography, University of Rhode Island, Narragansett, Rhode Island
}

(Manuscript received 13 November 2013, in final form 30 May 2014)

\begin{abstract}
Horizontal roll vortices, or rolls, are frequently observed in the hurricane boundary layer (HBL). Previous studies suggest that these rolls can be generated by the inflection point instability of the HBL flow. In this study we investigate the formation of rolls due to this mechanism in the axisymmetric HBL using a numerical approach that explicitly resolves rolls. The effects of mean HBL wind and stratification distributions on rolls are evaluated. We identify two important factors of the mean HBL wind that affect the characteristics of rolls. The dynamical HBL height affects the wavelength of rolls, and the magnitude of the mean wind shear affects the growth rate of rolls. As a result, under neutrally stratified HBL, the wavelength of rolls increases with the radius (out of the radius of maximum wind), while the growth rate of rolls decreases. The stratification also plays an important role in the generation of rolls. The stable stratification suppresses the growth of rolls because of the negative work done by the buoyancy force. Nonuniform stratification with a mixed layer has less suppressing effect on rolls. Rolls can trigger internal waves in the stably stratified layer, which have both vertically propagating and decaying properties. We derive analytical solutions for the internal waves, which relate the properties of the internal waves to the boundary layer rolls. We find the properties of the internal waves are affected by the mixed-layer height.
\end{abstract}

\section{Introduction}

Recent observations suggest that horizontal roll vortices, or rolls, frequently occur in the hurricane boundary layer (HBL). Most of these observations were obtained by Doppler radar from landfalling hurricanes (Wurman and Winslow 1998; Morrison et al. 2005; Lorsolo et al. 2008; Ellis and Businger 2010). In the radar observations, rolls are detected because they induce organized perturbations in the wind field. The wavelength of rolls estimated from the radar observations is highly variable, ranging from a few hundred meters to a few kilometers. For example, Lorsolo et al. (2008) observed rolls in Hurricanes Isabel (2003) and Frances (2004) with wavelengths ranging from 200 to $650 \mathrm{~m}$. Morrison et al. (2005) found the majority of rolls during the landfalls of four

¿ Denotes Open Access content.

Corresponding author address: Kun Gao, Graduate School of Oceanography, University of Rhode Island, 215 South Ferry Rd., Narragansett, RI 02882.

E-mail:kun_gao@my.uri.edu hurricanes had wavelength in the range $1-2 \mathrm{~km}$. Synthetic aperture radar observations identified rolls with wavelengths of 3-6 km (Katsaros et al. 2000). Zhang et al. (2008) provided the first in situ measurement of the rollinduced vertical fluxes in the HBL and showed that the rolls enhanced the total turbulent momentum and moisture fluxes in the HBL. However, the mixing effects of rolls are not represented in current hurricane models, which may limit their forecast accuracy. To represent the roll-induced vertical momentum and energy transports in hurricane numerical models, we must better understand their formation mechanism.

The formation mechanism and characteristics of rolls in the HBL were studied based on theoretical and numerical approaches. Foster (2005) applied linear and nonlinear analyses to the HBL momentum equations and argued that rolls are expected to be a common feature in the HBL. He demonstrated that the inflection points in the basic-state wind profiles cause the instability and lead to the generation of rolls. Such mechanism is analogous to the classical Ekman boundary layer instability (Faller 1965; Lilly 1966; Brown 1970, 1972). Since this type of instability is related to the inflection point in the boundary layer wind profile, it is commonly referred to as the 
inflection point instability. Wavelengths of the rolls estimated by Foster (2005) were similar to those observed by Morrison et al. (2005). Nolan (2005) used both a nonlinear axisymmetric model and a linear instability analysis approach to investigate the instability in the HBL. He also found that inflection points in the HBL wind profiles are responsible for the formation of rolls, which have wavelengths of 3-5 km. Zhu (2008) used the Large-Eddy Simulation version of the Weather Research and Forecasting model (WRF LES) nested within the WRF mesoscale model to simulate large eddies in a landfalling hurricane. He found roll-like perturbations existed in a statically stable boundary layer environment, suggesting these features were generated by the inflection point instability rather than the thermal instability. Nakanishi and Niino (2012) conducted an LES study at two different locations in the idealized HBL. By applying the EOF analysis to the model results, they identified rolls generated by the inflection point instability with spatial scales similar to those found by Foster (2005).

These previous studies investigated the rolls in neutral or near-neutral stratification (Foster 2005; Nolan 2005) or the rolls at specific locations within the HBL (Zhu 2008; Nakanishi and Niino 2012), but they did not systematically examine the conditions favorable for the formation of rolls by the inflection point instability and the impacts of the mean wind and stratification on the characteristics of rolls. In this study, we aim to gain a more comprehensive understanding of the formation of rolls under various HBL conditions and identify the important mean-flow factors affecting the characteristics of rolls. Specifically, this study addresses the following questions: What factors affect the spatial and temporal characteristics of the rolls generated by the inflection point instability? What are the spatial variations of the rolls in the HBL? What are the effects of stratification on the rolls? To answer these questions, we use a numerical approach that explicitly resolves the rolls in the HBL. We examine the formation and characteristics of rolls under various ambient environments by a set of idealized numerical experiments.

\section{Method}

\section{a. Modeling approach}

We assume that rolls can be separated from the largescale HBL flow because of their small spatial scale. Based on this assumption, we decompose the HBL flow variables into the mean and perturbations, as $a=\bar{a}+a^{\prime}$, where $a$ can represent the velocity vector, potential temperature, etc. Similar flow decomposition approach was applied by Ginis et al. (2004) and Foster (2005) for

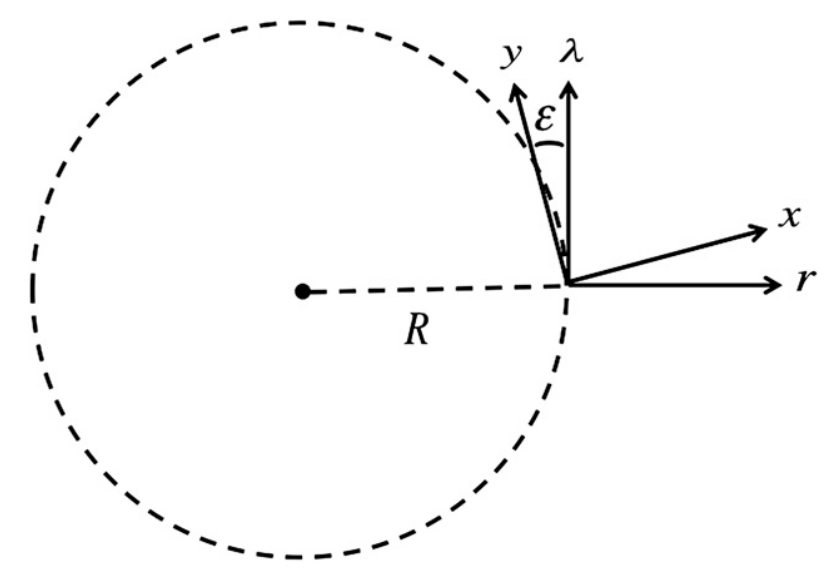

FIG. 1. Diagram illustrating the cylindrical coordinate system $(r, \lambda, z)$ for the mean flow and local Cartesian coordinate system $(x, y, z)$ for rolls at radius $r=R$ ( $z$ axis is not shown). Parameter $\varepsilon$ is the angle between the along-roll direction $y$ and the azimuthal direction $\lambda$. Positive $\varepsilon$ means the along-roll direction is to the left of the azimuthal direction.

studying boundary layer eddies or rolls under hurricane condition. In this study, the mean component represents the large-scale HBL flow, which consists of the primary cyclonic circulation and the secondary circulation induced by surface friction; the perturbations represent the rolls, which are the small-scale features generated by the inflection point instability of the mean HBL flow.

For simplicity, the mean HBL flow is assumed axisymmetric and therefore can be described in a cylindrical coordinate system $(r, \lambda, z)$. Observations suggest that rolls have quasi-two-dimensional structures and they are elongated approximately in the mean wind direction. We use a local Cartesian coordinate system $(x, y, z)$ for the rolls at large radius in the hurricane (radius larger than the radius of the maximum wind). The system $(x, y, z)$ is set up in such a way that the $y$ axis is parallel to the direction in which the rolls are aligned. We will refer to the $y$ axis ( $x$ axis) as the along-roll axis (cross-roll axis). The along-roll variations of the perturbations are assumed negligible; that is, $\partial a^{\prime} / \partial y=0$, where $a^{\prime}$ represents the variables associated with roll motions. This assumption allows for reducing the three-dimensional equations for the rolls into two-dimensional equations written in an $x-z$ plane. To distinguish the velocity components projected onto the two different coordinate systems, we use uppercase letters $(U, V, W)$ to represent the wind components in the cylindrical coordinate system and lowercase letters $(u, v, w)$ to represent the wind components in the local Cartesian coordinate system. If the angle between the along-roll direction $y$ and the azimuthal direction $\lambda$ is defined as $\varepsilon$ (Fig. 1), the wind components in the two coordinate systems can be transformed as follows: 


$$
\left[\begin{array}{l}
u \\
v
\end{array}\right]=\left[\begin{array}{ll}
\cos \varepsilon & \sin \varepsilon \\
-\sin \varepsilon & \cos \varepsilon
\end{array}\right]\left[\begin{array}{l}
U \\
V
\end{array}\right] \text { and } w=W
$$

The complete sets of equations for the mean flow and rolls are presented in the appendix. For the purpose of this study, the governing equations for the mean flow and rolls are further simplified. We consider the evolution of the rolls to take place in two phases: the linear phase and the nonlinear phase. During the linear phase, rolls are formed by the instability of the mean flow and have the following characteristics: 1) roll velocities grow exponentially with time, but remain at least one order smaller than the mean winds, and thus the nonlinear terms in their governing equations are negligible; and 2) the averaged fluxes induced by rolls are too weak to cause any significant modifications to the mean flow, and therefore we can assume the mean flow remains unchanged during the linear phase. The evolution of rolls enters the nonlinear phase after roll velocities reach sufficiently large magnitude (comparable to the mean winds), during which the nonlinear effects and the roll-induced fluxes are important. In this paper, we focus on the generation and initial evolution of rolls and therefore only consider the linear phase. Thus some terms in the governing equations of the mean flow and rolls can be neglected, as discussed below.

\section{b. The basic-state HBL flow}

The basic-state HBL flow is used as the background environment for the formation of rolls. The basic-state HBL wind profiles are obtained by resolving the steadystate mean wind equations, neglecting time dependency and roll-induced forcing terms in (A4)-(A6). While the basic-state potential temperature profiles are prescribed analytically. We assume that the basic-state HBL wind and potential temperature profiles remain unchanged over time during the linear phase of rolls.

The governing equations for the basic-state HBL winds are

$$
\begin{gathered}
\bar{U} \frac{\partial \bar{U}}{\partial r}+\bar{W} \frac{\partial \bar{U}}{\partial z}-\frac{\bar{V}^{2}}{r}-f \bar{V}=-\frac{1}{\rho_{0}} \frac{\partial \bar{P}}{\partial r}+\bar{K}_{m} \frac{\partial^{2} \bar{U}}{\partial z^{2}}, \\
\bar{U} \frac{\partial \bar{V}}{\partial r}+\bar{W} \frac{\partial \bar{V}}{\partial z}+\frac{\bar{U} \bar{V}}{r}+f \bar{U}=\bar{K}_{m} \frac{\partial^{2} \bar{V}}{\partial z^{2}}, \quad \text { and } \\
\frac{\partial \bar{U}}{\partial r}+\frac{\bar{U}}{r}+\frac{\partial \bar{W}}{\partial z}=0,
\end{gathered}
$$

where $\bar{U}, \bar{V}$, and $\bar{W}$ are the mean wind components in the radial, azimuthal, and vertical directions, respectively, and $\bar{K}_{m}$ is the mean turbulent viscosity.

The hurricane is assumed to be on the $f$ plane centered at $20^{\circ} \mathrm{N}$. At the upper boundary $(z=H$, where $H$ is the

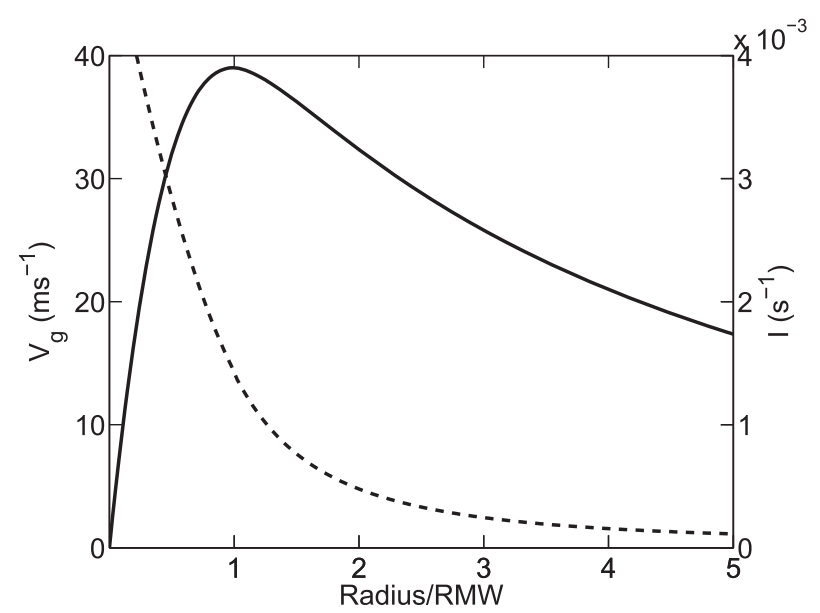

FIG. 2. Gradient wind (solid line) and inertial stability parameter (dashed line) as functions of radius (normalized by the radius of maximum wind).

vertical extent of the atmospheric layer that we consider), we assume the wind is under gradient wind balance; that is, $\bar{V}=V_{g}$ and $\bar{U}=0$, where $V_{g}$ is the gradient wind, satisfying

$$
\frac{V_{g}^{2}}{r}-f V_{g}=-\frac{1}{\rho_{0}} \frac{\partial \bar{P}}{\partial r} .
$$

We use the Holland (1980) parametric model to specify the radial distribution of $V_{g}$ at the upper boundary and assume the pressure gradient force does not vary vertically in the shallow atmosphere layer that we consider. The radial $V_{g}$ profile that we use has a maximum wind speed of $39 \mathrm{~m} \mathrm{~s}^{-1}$, a radius of maximum wind (RMW) of $40 \mathrm{~km}$, and a parameter $B$ of 1.3 (parameter $B$ controls how rapidly $V_{g}$ decreases with radius). This profile represents a generic hurricane and has been used in other HBL studies (Kepert 2001, 2012; Kepert and Wang 2001, hereafter KW01; Foster 2005, 2009). The distribution of $V_{g}$ inside of the RMW is modified following the formulation used by KW01. Figure 2 shows the radial distribution of $V_{g}$ and the inertial stability parameter for the storm that we consider in this study. The inertial stability parameter is given by $I=\sqrt{\left(f+2 \bar{V}_{g} / r\right)\left(f+\bar{V}_{g} / r+\partial \bar{V}_{g} / \partial r\right)}($ Kepert 2001). The vertical distribution of the velocity and turbulent viscosity near the lower boundary (from the surface to the lowest model level) is determined using the MoninObukhov similarity theory as described in Ginis et al. (2004). The only difference from Ginis et al. (2004) is that we calculate the roughness length using the formulation proposed by Moon et al. (2007). This formulation limits the drag coefficient below 0.003 at high wind speed, which is within the error bars estimated by Powell et al. (2003) 
based on in situ observations and consistent with the results from the laboratory experiments conducted by Donelan et al. (2004).

One critical parameter that affects the basic-state HBL wind distribution is $\bar{K}_{m}$. We calculate $\bar{K}_{m}$ using a first-order scheme as recommended by Zhang and Drennan (2012) and Kepert (2012). In this scheme, called the Louis scheme following Kepert (2012), $\bar{K}_{m}$ is parameterized in the form

$$
\bar{K}_{m}=l^{2} S f(\mathrm{Ri}),
$$

where $l$ is the mixing length, $S$ is the magnitude of the vertical wind shear, and $\mathrm{Ri}$ is the gradient Richardson number. We apply the neutral version of the Louis scheme; that is, $f(\mathrm{Ri})=1$. The mixing length has the form suggested by Blackadar (1962), written as

$$
\frac{1}{l}=\frac{1}{\kappa z}+\frac{1}{l_{\infty}}
$$

where $\kappa$ is the von Kármán constant $(\kappa=0.4)$ and $l_{\infty}$ is asymptotic mixing length.

The model described above is called the basic-state HBL model. Figure 3 shows an example of the basicstate HBL wind solution with the choice of $l_{\infty}=80 \mathrm{~m}$. The overall structure of the boundary layer is consistent with observations (Zhang et al. 2011) and similar to the simulation in Kepert (2012) using the same $l_{\infty}$ [see Fig. 3 in Kepert (2012)]. Representative wind profiles at selected locations, 1RMW $(r=40 \mathrm{~km}), 2 \mathrm{RMW}(r=80 \mathrm{~km})$, and 3RMW $(r=120 \mathrm{~km})$, are shown in Fig. 4. Both the radial and azimuthal wind profiles have inflection points (only the inflection points in the radial wind profiles are marked in Fig. 4). The vertical wind shear reaches extreme value at the inflection point. Compared with the inflection point in the azimuthal wind profile, the inflection point in the radial wind profile has considerably larger wind shear.

\section{c. Equations for rolls in the linear phase}

The nonlinear terms in the roll equations are negligible during the linear phase of rolls. Sensitivity experiments suggest that the Coriolis and curvature terms in the roll equations do not affect the solutions in any significant way and thus can also be neglected, which is consistent with Foster (2005). By neglecting these terms, we decouple the along-roll velocity component $v^{\prime}$ from the cross-roll motions $u^{\prime}$ and $w^{\prime}$. This effectively filters out the parallel instability, which is another type of dynamical instability that can operate in Ekman-like boundary layer flow (Lilly 1966). Given these considerations, the equations governing the linear-phase rolls are
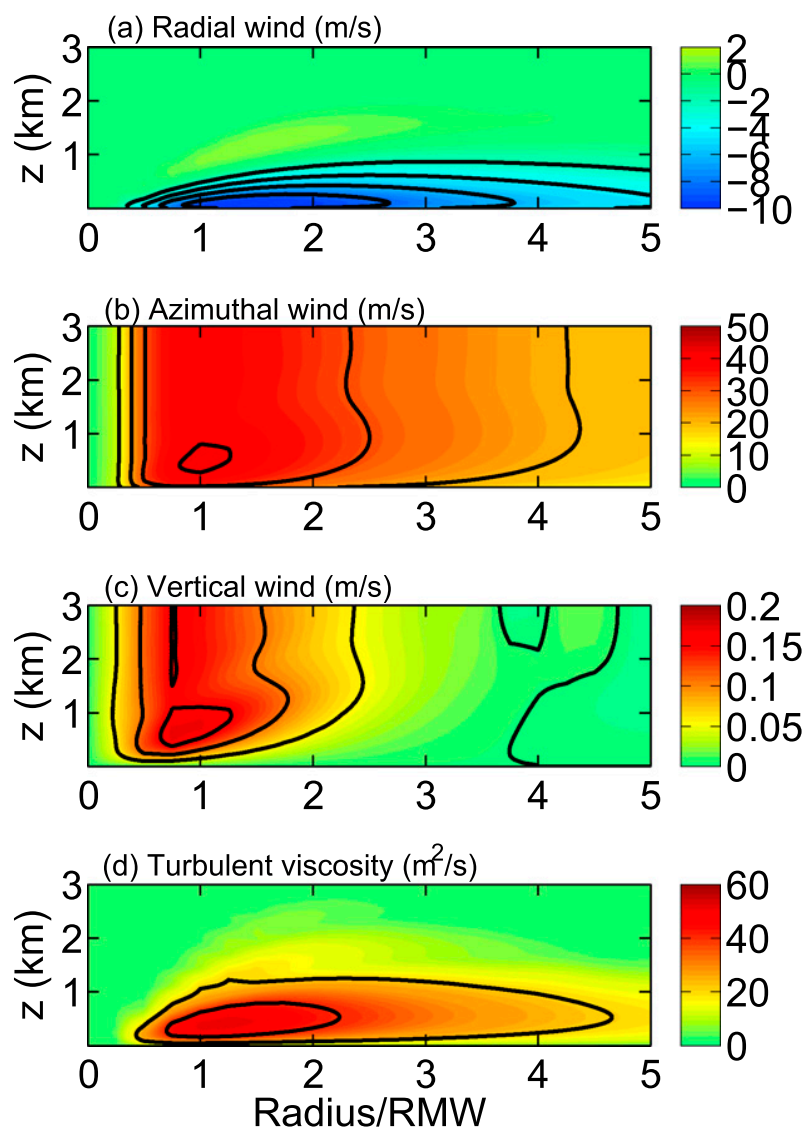

FIG. 3. Height-radius distribution of the basic-state hurricane boundary layer variables with $l_{\infty}=80 \mathrm{~m}$. Black contours are multiples of (a) -2 , (b) 10 , (c) 0.05 , and (d) $20 \mathrm{~m}^{2} \mathrm{~s}^{-1}$.

$$
\begin{aligned}
\frac{\partial \eta^{\prime}}{\partial t} & =-\bar{u} \frac{\partial \eta^{\prime}}{\partial x}+\frac{\partial \psi^{\prime}}{\partial x} \frac{\partial^{2} \bar{u}}{\partial z^{2}}+\frac{g}{\theta_{0}} \frac{\partial \theta^{\prime}}{\partial x}+\bar{K}_{m} \nabla^{2} \eta^{\prime}, \\
\frac{\partial v^{\prime}}{\partial t} & =-\bar{u} \frac{\partial v^{\prime}}{\partial x}-\frac{\partial \psi^{\prime}}{\partial x} \frac{\partial \bar{v}}{\partial z}+\bar{K}_{m} \nabla^{2} v^{\prime}, \\
\frac{\partial \theta^{\prime}}{\partial t} & =-\bar{u} \frac{\partial \theta^{\prime}}{\partial x}-\frac{\partial \psi^{\prime}}{\partial x} \frac{\partial \bar{\theta}}{\partial z}+\bar{K}_{h} \nabla^{2} \theta^{\prime}, \quad \text { and } \\
\eta^{\prime} & =\nabla^{2} \psi^{\prime},
\end{aligned}
$$

where $\nabla^{2}=\partial^{2} / \partial x^{2}+\partial^{2} \partial z^{2}$. Variables with the prime are associated with roll motions: $\psi^{\prime}$ is the streamfunction (cross-roll velocity $u^{\prime}=-\partial \psi^{\prime} / \partial z$, vertical velocity $\left.w^{\prime}=\partial \psi^{\prime} / \partial x\right), \eta^{\prime}$ is the along-roll vorticity $\left(\eta^{\prime}=\partial w^{\prime} / \partial x-\partial u^{\prime} / \partial z\right), v^{\prime}$ is the along-roll velocity, and $\theta^{\prime}$ is the potential temperature perturbations. Variables with the overbar are associated with the mean flow: $\bar{u}$ and $\bar{v}$ are the mean winds in the cross-roll and along-roll directions respectively, $\bar{\theta}$ is the mean potential temperature, and $\bar{K}_{m}$ and $\bar{K}_{h}$ are the mean turbulent viscosity and diffusivity, respectively. The above set of equations 

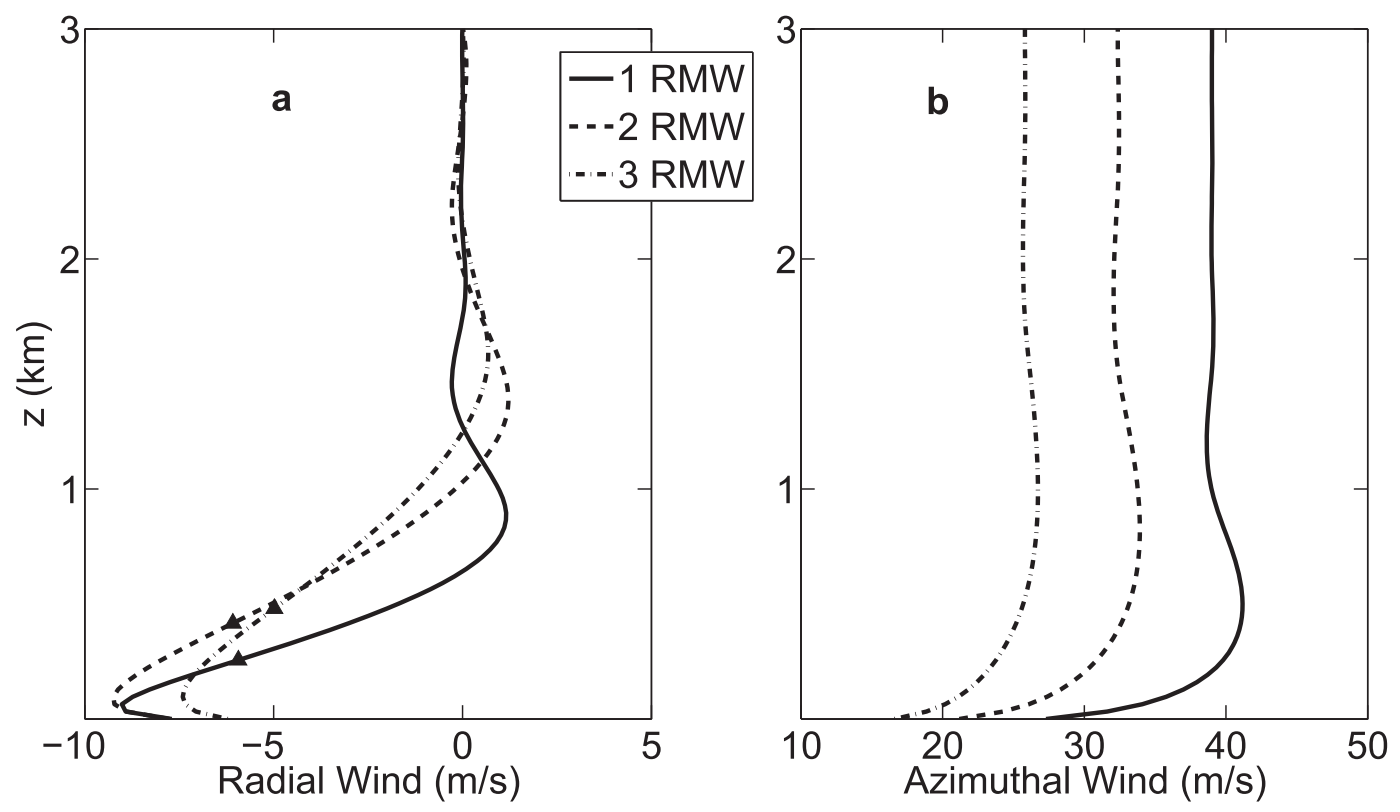

FIG. 4. Vertical profiles of (a) radial wind and (b) azimuthal wind at 1RMW, 2RMW, and 3RMW in the hurricane boundary layer with $l_{\infty}=80 \mathrm{~m}$. The inflection points with the largest radial wind shear are indicated by the black triangles in (a).

describes the evolution of rolls during the linear phase at a specific location in the HBL. The mean-flow parameters $\left(\bar{u}, \bar{v}, \bar{\theta}, \bar{K}_{m}\right.$, and $\left.\bar{K}_{h}\right)$ are given by the basic-state HBL flow. For simplicity, we assume $\bar{K}_{h}$ is equal to $\bar{K}_{m}$.

Periodic conditions are applied at the lateral boundaries. At the upper and lower boundaries, we set $\eta^{\prime}=$ $\theta^{\prime}=w^{\prime}=0\left(\eta^{\prime}=w^{\prime}=0\right.$ implies $\left.\partial u^{\prime} / \partial z=0\right)$ and $\partial v^{\prime} / \partial z=0$. We also tested the no-slip conditions $\left(u^{\prime}=v^{\prime}=0\right)$ and found that away from the lower and upper boundaries the structure of rolls is not affected. An infinitesimal perturbation in the vorticity field is turned on as the initial condition for the perturbations. The model described above is referred to as the Single-Grid RollResolving Model (SRM). It resolves rolls based on the mean-flow profiles at a single grid of the basic-state HBL model.

\section{d. Experimental design}

Three groups of experiments are designed to investigate the impacts of the basic-state wind and stratification on the formation and characteristics of rolls. In the numerical experiments presented below, the SRM is embedded at every grid point of the basic-state HBL model from 1RMW to 3RMW. The horizontal and vertical resolutions and the domain sizes for both models are listed in Table 1. The calculations in each experiment are done in two steps. During the first step, the basic-state HBL model resolves (1)-(3) to derive the basic-state wind distribution. During the second step, the SRM resolves (7)-(10) based on the basic-state wind profiles and the prescribed potential temperature profiles. Both theoretical analysis (Foster 2005) and three-dimensional LES study (Nakanishi and Niino 2012) suggested that the angle between the along-roll axis and the azimuthal wind is relatively small. Therefore, for simplicity we assume that $\varepsilon=0$-that is, the along-roll (cross roll) axis is in the local azimuthal (radial) wind direction $(\bar{u}=\bar{U}$ and $\bar{v}=\bar{V}$ )—in all groups of experiments.

Group $\mathrm{N}$ is designed to investigate the effect of basicstate HBL winds. In this group of experiments, we neglect the effect of stratification on the rolls by assuming neutral stratification (i.e., $\partial \bar{\theta} / \partial z=0$ in the entire atmosphere layer that we consider). Similar to the modeling results of Kepert (2012), our basic-state HBL model simulations indicate that an increase of $l_{\infty}$ results in an increase of the boundary layer height and a decrease of the wind shear. Therefore, we use different values of $l_{\infty}$ (listed in Table 2) to vary the distribution of basic-state HBL winds in group $\mathrm{N}$.

TABLE 1. Numerical parameters for the basic-state HBL model and the SRM.

\begin{tabular}{lcl}
\hline \hline & Basic-state HBL model & \multicolumn{1}{c}{ SRM } \\
\hline Vertical extent & $3.008 \mathrm{~km}$ & $3.008 \mathrm{~km}$ \\
Horizontal extent & $1000 \mathrm{~km}$ & $16.384 \mathrm{~km}$ \\
Vertical resolution & $32 \mathrm{~m}$ & $32 \mathrm{~m}$ \\
Horizontal resolution & $10 \mathrm{~km}$ & $32 \mathrm{~m}$ \\
\hline
\end{tabular}


TABLE 2. Values of asymptotic mixing length used in group $\mathrm{N}$. Group $\mathrm{N}$ is designed to investigate the effect of basic-state HBL wind distribution on rolls. Neutral stratification is applied in group $\mathrm{N}$ and distribution of the basic-state HBL winds is varied by changing $l_{\infty}$.

\begin{tabular}{cc}
\hline \hline Experiment name & $l_{\infty}(\mathrm{m})$ \\
\hline N1 & 10 \\
N2 & 20 \\
N3 & 40 \\
N4 & 60 \\
N5 & 80 \\
\hline
\end{tabular}

Groups $\mathrm{S}$ and $\mathrm{M}$ are designed to investigate the effect of stratification on roll formation and characteristics. The basic-state HBL winds used in groups $\mathrm{S}$ and $\mathrm{M}$ are as in experiment $\mathrm{N} 3\left(l_{\infty}=40 \mathrm{~m}\right)$ in group $\mathrm{N}$, but the potential temperature profiles are varied. GPS dropsondes measurements collected in hurricanes (Zhang et al. 2011) suggest that the potential temperature profiles in the HBL usually contain a stably stratified layer $(d \theta / d z>0)$, a relatively well-mixed layer $(d \theta / d z \sim 0)$, and a nearsurface unstably stratified $(d \theta / d z<0)$ layer (Fig. 5). In groups $\mathrm{S}$ and $\mathrm{M}$, two different types of idealized potential temperature profiles are used to investigate the effects of stratification. Since we are only concerned with the inflection point instability, we do not consider the unstably stratified layer in the potential temperature profiles to exclude the convective instability. Group $\mathrm{S}$ is aimed to investigate the effect of stable stratification. Potential temperature profiles with constant lapse rate $(\partial \bar{\theta} / \partial z)$ are used in group $\mathrm{S}$, and the lapse rate is varied (Table 3 ). There is no mixed layer in the potential temperature profiles used in group $\mathrm{S}$. The same potential temperature profile is applied at all the locations from 1RMW to 3RMW. Group $M$ focuses on the effect of the mixed layer. In contrast to group S, we use potential temperature profile that contains a well-mixed layer $(\partial \bar{\theta} / \partial z=0)$ and a stably stratified layer above in group $\mathrm{M}$, as shown in Fig. 6. The lapse rate in the stably stratified layer is fixed at $5 \mathrm{~K} \mathrm{~km}^{-1}$ and the mixed-layer height $z_{i}$ is varied from approximately 200 to $600 \mathrm{~m}$ (Table 4). The value of the lapse rate and the range of the mixed-layer heights that we use in group $\mathrm{M}$ are consistent with those observed in hurricane conditions (Fig. 5).

\section{Effect of mean wind on rolls}

\section{a. Basic structure of rolls}

Rolls are formed in all experiments of group $\mathrm{N}$ owing to the inflection point instability. They have similar structures, but their wavelengths and growth rates vary with the changes of the mean wind profiles. The typical structure of rolls is shown in Fig. 7. Since rolls in the linear phase grow exponentially with time, the variables shown in Fig. 7 are normalized to remove the timedependent part. The length scale $\delta$ that we use to nondimensionalize the horizontal and vertical axes is the dynamical HBL height scale suggested by Kepert (2001). It is defined as $\delta=\sqrt{2 K / I}$, where $I$ is defined above and $K$ is the turbulent viscosity. Here we use the maximum value in the $\bar{K}_{m}$ profile to calculate $\delta$. The rolls shown in Fig. 7 have similar structure to those derived by Foster (2005). One noticeable feature is the tilted streamlines near the surface $(z / \delta<3)$. The tilted streamlines create asymmetry in the distribution of $w^{\prime} u^{\prime}$ (the product of vertical velocity and cross-roll velocity; Fig. 7d): $w^{\prime} u^{\prime}$ is mostly negative near the surface. While at upper levels $(z / \delta>3)$, the streamlines and $w^{\prime} u^{\prime}$ are more vertically symmetric.
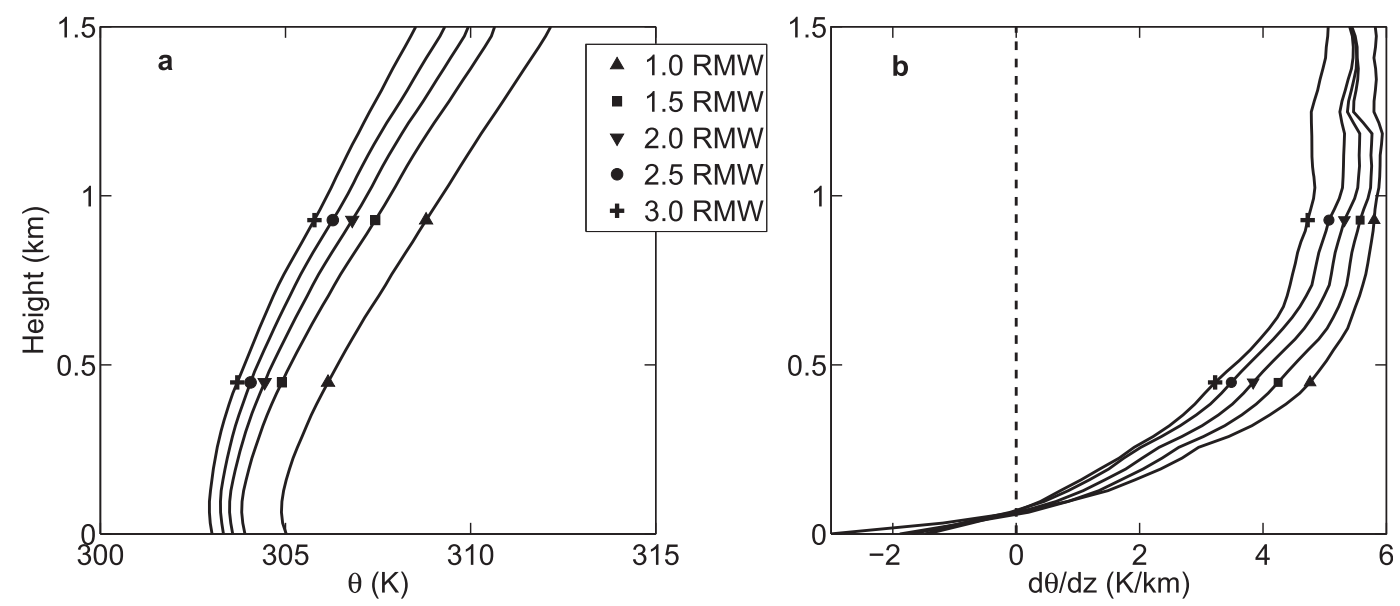

FIG. 5. Vertical profiles of (a) potential temperature and (b) lapse rate in the hurricane boundary layer from a composite dataset based on GPS dropsondes measurements (Zhang et al. 2011). The approximate locations of these profiles are indicated $(\mathrm{RMW}=40 \mathrm{~km})$. 
TABLE 3. Values of lapse rate used in group S. Group $\mathrm{S}$ is used to investigate the effect of stable stratification on rolls. The basic-state HBL winds used in group S are as in experiment N3 $\left(l_{\infty}=40 \mathrm{~m}\right)$ in group $\mathrm{N}$. Uniform stratification is applied in group $\mathrm{S}$ and the lapse rate of the potential temperature profiles is varied. There is no mixed layer in the potential temperature profiles used in this group of experiments. The same potential temperature profile is applied at all locations in each experiment.

\begin{tabular}{cc}
\hline \hline Experiment name & $\partial \bar{\theta} / \partial z\left(\mathrm{~K} \mathrm{~km}^{-1}\right)$ \\
\hline S1 & 1 \\
S2 & 2 \\
S3 & 3 \\
S4 & 4 \\
S5 & 5 \\
\hline
\end{tabular}

Next, we consider the kinetic energy budget for rolls to help us better understand the role of wind shear and stratification in the roll formation. Here we define the kinetic energy of rolls as $e^{\prime}=0.5\left(u^{\prime 2}+w^{\prime 2}\right)$; $v^{\prime}$ is excluded from the energy budget consideration because it has no influence on the cross-roll overturning circulation $\left(u^{\prime}, w^{\prime}\right)$. The equation for $e^{\prime}$ is derived from (7)-(10) and can be written as

$\frac{d}{d t} \int \overline{e^{\prime}} d z=\int\left(-\overline{w^{\prime} u^{\prime}} \frac{\partial \bar{u}}{\partial z}+\frac{g}{\theta_{0}} \overline{w^{\prime} \theta^{\prime}}-\bar{K}_{m} \overline{\eta^{\prime 2}}\right) d z$,

where $\overline{e^{\prime}}$ is the cross-roll-averaged kinetic energy and $\int d z$ denotes the vertical integration over the atmospheric layer that we consider. The three terms on the right-hand side represent the shear production, buoyancy work, and the kinetic energy dissipation, respectively. In neutral stratification, the only energy source for the rolls is the shear production. Figure 8 shows the vertical distribution of the shear production for the rolls in Fig. 7. Such distribution is representative for all rolls in group $\mathrm{N}$. The mean wind shear $(\partial \bar{u} / \partial z)$ reaches the maximum value at the inflection point. Correspondingly, the averaged crossroll momentum flux $\left(\overline{w^{\prime} u^{\prime}}\right)$ also reaches maximum value at the height of the inflection point. The tilted nearsurface streamlines make the averaged momentum flux negative (opposite to the sign of the mean wind shear) and thus make the shear production term positive. Therefore, we can conclude that the near-surface tilted streamlines are necessary for rolls to extract the kinetic energy from the mean wind.

\section{b. Factors affecting growth rate and wavelength of rolls}

We next explore the characteristics of rolls, specifically their wavelength and growth rate, and identify mean wind factors that affect these characteristics. The

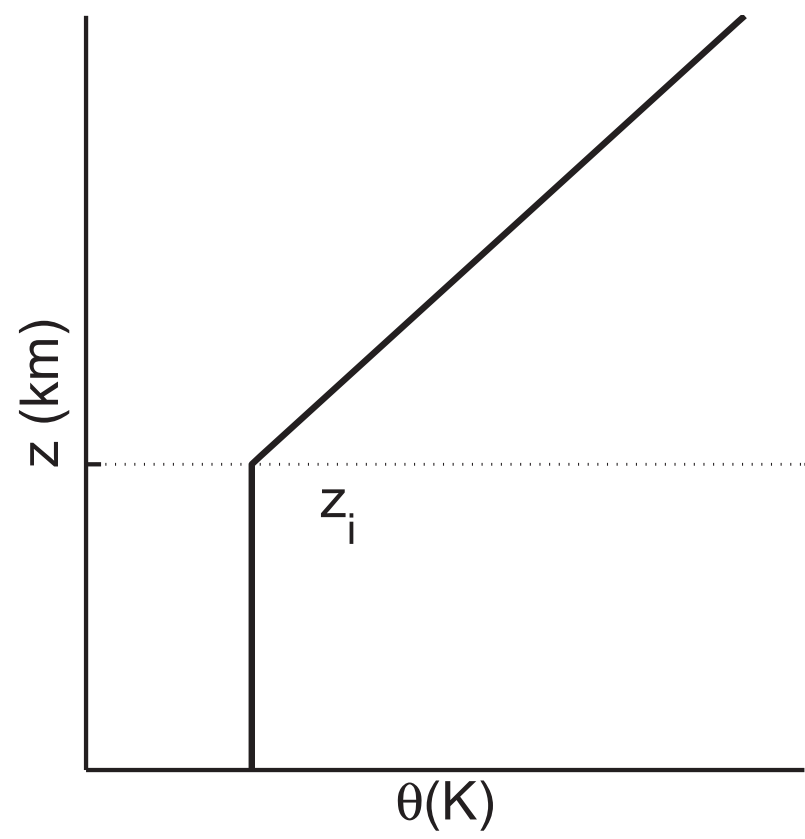

FIG. 6. Idealized potential temperature profile used in group M. The profile consists of two layers: the mixed layer and the stably stratified layer. The potential temperature is vertically uniform in the mixed layer and increases linearly with height in the stably stratified layer. The height of the mixed layer is indicated by the dotted line.

SRM solutions indicate roll variables are in the form of normal mode. For example, the vertical velocity can be written as $w^{\prime}=\hat{w}(z) \operatorname{expi}(k x-\omega t)$, where $k$ is the horizontal wavenumber, $\hat{w}(z)$ and $\omega$ are complex, $\omega=\omega_{r}+i \omega_{i}, \omega_{r}$ is the angular frequency, and $\omega_{i}$ is the growth rate. We derive the wavelength of rolls $(2 \pi / k)$ by applying Fourier analysis on the horizontal distribution of $w^{\prime}$. The wavelength corresponding to the peak spectral density is considered as the wavelength of rolls. In the linear phase, the wavelength of rolls is vertically coherent and does not vary with time. The growth rate is

TABLE 4. Values of mixed-layer height used in group M. Group $\mathrm{M}$ is used to investigate the effect of the mixed layer on rolls. The basic-state $\mathrm{HBL}$ winds used in group $\mathrm{M}$ are as in experiment N3 $\left(l_{\infty}=40 \mathrm{~m}\right)$ in group $\mathrm{N}$. In contrast to group S, nonuniform stratification containing a mixed layer $(\partial \bar{\theta} / \partial z=0)$ and a stably stratified layer $\left(\partial \bar{\theta} / \partial z=5 \mathrm{~K} \mathrm{~km}^{-1}\right)$ is used in group M. The same potential temperature profile is applied at all locations in each experiment.

\begin{tabular}{cc}
\hline \hline Experiment name & $z_{i}(\mathrm{~m})$ \\
\hline M1 & 192 \\
M2 & 288 \\
M3 & 384 \\
M4 & 480 \\
M5 & 576 \\
\hline
\end{tabular}



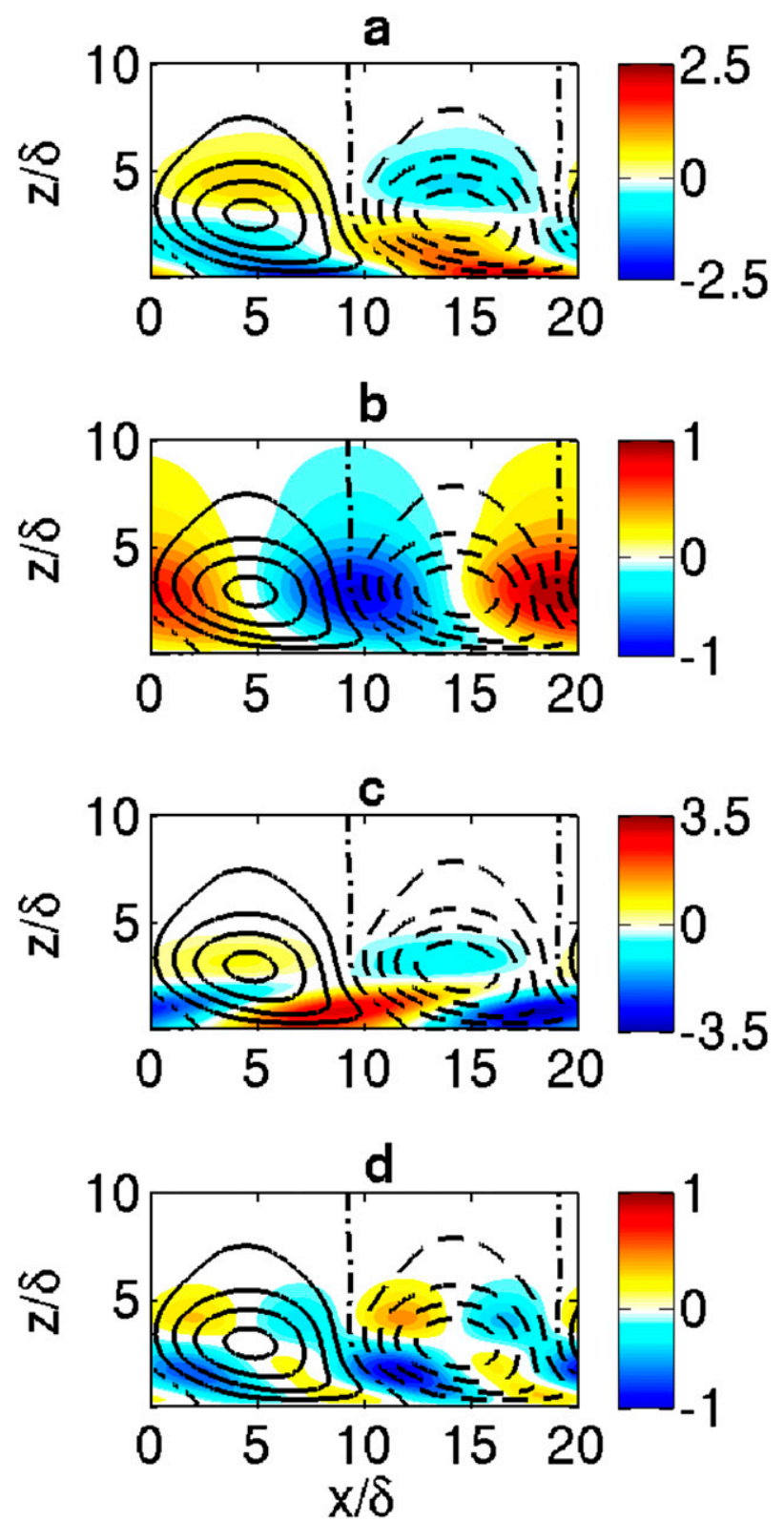

FIG. 7. Typical structure of rolls under neutral stratification. The colored backgrounds represent fields for (a) $u^{\prime}$, (b) $w^{\prime}$, (c) $v^{\prime}$, and (d) $w^{\prime} u^{\prime}$. The three velocity components are nondimensionalized by the maximum value of $w^{\prime} ; w^{\prime} u^{\prime}$ is nondimensionalized by its own maximum value. The $x$ and $z$ axes are nondimensionalized by $\delta$. Contours are streamlines: solid (dashed) contours represent clockwise (counterclockwise) circulations. The rolls shown are at RMW in experiment N3 in group N with $\delta=200 \mathrm{~m}$.

estimated from the time series of the domain-averaged kinetic energy, using $\omega_{i}=0.5 d \log \left(\left\langle e^{\prime}\right\rangle\right) / d t$, where $\left\langle e^{\prime}\right\rangle$ is kinetic energy averaged over the entire SRM domain.

Figure 9 shows the wavelength of rolls and $\delta$ from all experiments in group $\mathrm{N}$. The wavelength of rolls increases with radius, which is consistent with the theoretical study of Foster (2005). The increase of roll wavelength is associated with the increase of $\delta$ (Fig. 9b). The ratios of the roll wavelength to $\delta$ in all experiments are in the range of 15-20. According to our basic-state HBL wind simulations, larger $\delta$ means that the shear layer (the layer in which the wind shear $\partial \bar{u} / \partial z$ is relatively large) is higher. Since the rolls gain the kinetic energy within the shear layer, the basic-state wind profiles with larger $\delta$ favor rolls with larger vertical and horizontal extents. This explains why the wavelength of rolls becomes larger with increasing radius.

Figure 10 shows the growth rate of rolls and the bulk wind shear from all experiments in group N. The bulk wind shear represents the magnitude of mean wind shear and is defined as $\bar{u}_{\max } / \delta$, where $\bar{u}_{\max }$ is the maximum cross-roll mean wind. The growth rate of rolls decreases with radius (Fig. 10a) and it is closely related to the bulk shear (Fig. 10b). This is because the mean wind shear is the only energy source for the rolls in the neutrally stratified HBL. Because of the decrease of the radial wind speed and the increase of the boundary layer height, the magnitude of the wind shear decreases with radius. As a result, the growth rate of rolls decreases with radius.

\section{Effects of stratification on rolls \\ a. Effect of the stable stratification}

The growth rates of rolls in the group $\mathrm{S}$ experiments are shown in Fig. 11a. The major effect caused by the stable stratification is the reduction the growth rate of rolls. At a given location, the growth rate decreases with increasing lapse rate. When the stratification is sufficiently strong, the growth rate can be reduced to zero, which means the inflection point instability is completely suppressed. We find that the classic Ri criterion ${ }^{1}$ (Miles 1961) can be used to determine whether the inflection point instability can operate under stable stratification. $\mathrm{Ri}$ is defined as $\mathrm{Ri}=N^{2} /(\partial \bar{u} / \partial z)^{2}$, where $N^{2}=\left(g / \theta_{0}\right)(\partial \bar{\theta} / \partial z)$. Since $N^{2}$ is a constant in group $\mathrm{S}$, Ri reaches minimum at the inflection point where the wind shear reaches maximum. The minimum $\mathrm{Ri}$ at each location is plotted in Fig. 11b. As seen in Fig. 11, rolls can only be generated when minimum $\mathrm{Ri}$ is less than 0.25 , while rolls cannot be generated when the minimum $\mathrm{Ri}$ is greater than 0.25 .

\footnotetext{
${ }^{1}$ The Richardson number criterion states that for a stratified shear flow, the necessary (but not sufficient) condition for the flow to be unstable is that the minimum $\mathrm{Ri}$ is less than 0.25 , and the sufficient (but not necessary) condition for the flow to become stable is that the minimum $\mathrm{Ri}$ is greater than 0.25 .
} 

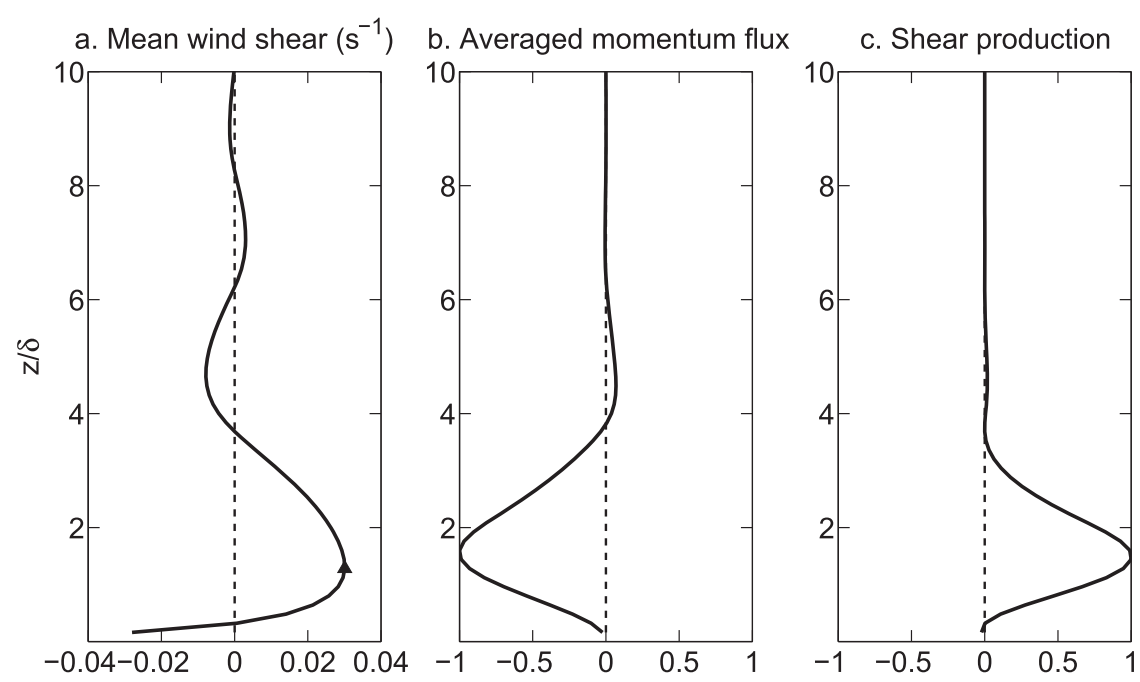

FIG. 8. Typical distributions of (a) $\partial \bar{u} / \partial z$, (b) $\overline{w^{\prime} u^{\prime}}$, and (c) $-\left(\overline{w^{\prime} u^{\prime}}\right)(\partial \bar{u} / \partial z)$ for rolls at RMW in experiment N3, as shown in Fig. 6. The averaged cross-roll momentum flux and shear production shown are normalized by their maximum values. The vertical axis is nondimensionalized by $\delta(\delta=200 \mathrm{~m})$. The height of the inflection point is indicated by the black triangle in (a).

The effect of stable stratification can be explained by the buoyancy work in the kinetic energy budget equation [see (11)]. The correlation between $w^{\prime}$ and $\theta^{\prime}$ determines the sign of the buoyancy work. Typical distributions of $\theta^{\prime}$, as well as the product of $w^{\prime}$ and $\theta^{\prime}\left(w^{\prime} \theta^{\prime}\right)$ are shown in Fig. 12. In the lower part of rolls $w^{\prime}$ and $\theta^{\prime}$ are negatively correlated, which indicates that the rolls tend to move heavier air parcels upward and lighter air parcels downward. As a result, the buoyancy work term in (11) is negative, causing the reduction of the growth rate. Typical vertical distributions of the buoyancy work and the shear production in group $\mathrm{S}$ are shown in Fig. 13. As the ambient lapse rate increases, the ratio of buoyancy work to shear production also increases (not shown). This means stronger stratification results in stronger negative buoyancy work, and thus explains why the growth rate of rolls decreases with increasing stratification. When the strength of stable stratification reaches a critical value (indicated by the critical Ri, 0.25 ), rolls cannot be generated (i.e., the inflection point instability is completely suppressed).

\section{b. Effect of the mixed layer}

Figure 14a shows the growth rate of rolls in group M. Overall, the mixed layer relaxes the suppressing effect of stratification on the inflection point instability. In the presence of a mixed layer, rolls can be formed at the locations where the inflection point instability is completely suppressed without the mixed layer (e.g., experiment S5 in group S). The roll growth rate increases with increasing $z_{i}$, suggesting the buoyancy work weakens as $z_{i}$ increases. When the mixed layer is sufficiently high (e.g., experiments M5), the growth rate of rolls is almost the same as in the experiment when stratification is not considered (experiment $\mathrm{N} 3$ in group $\mathrm{N}$ ).

The typical structure of rolls in the group $\mathrm{M}$ experiments is shown in Fig. 15. The near-surface part of the rolls appears to be similar to those in groups $\mathrm{N}$ (Fig. 7)

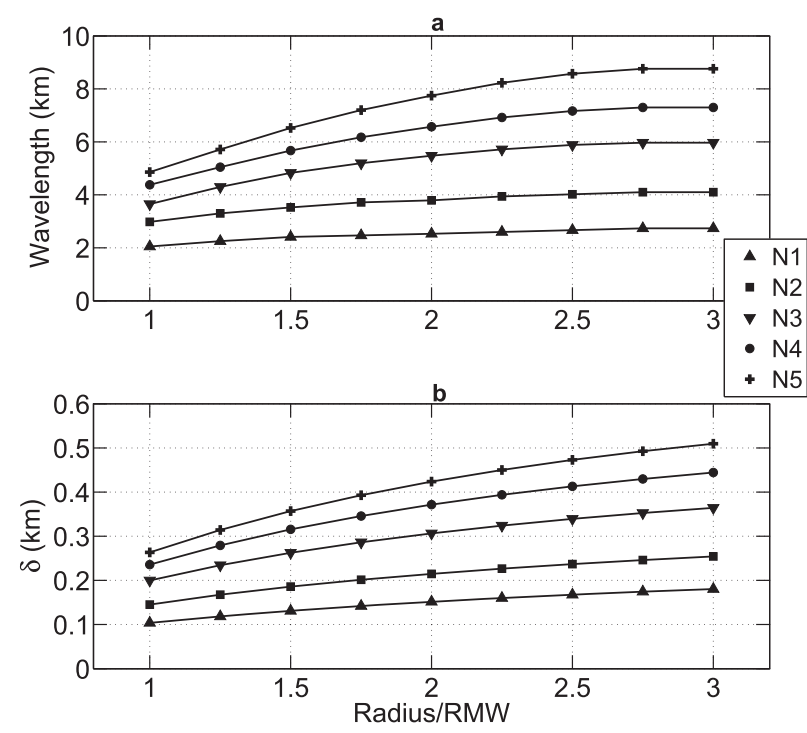

FIG. 9. (a) Wavelength of rolls and (b) $\delta$ as functions of radius in group $\mathrm{N}$. 

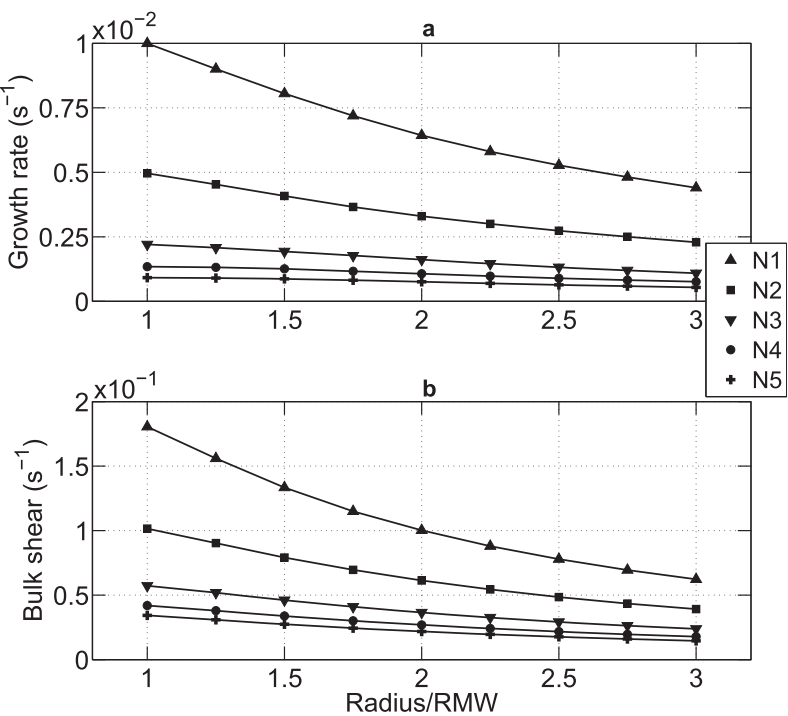

FIG. 10. (a) Growth rate of rolls and (b) the bulk shear as functions of radius in group $\mathrm{N}$.

and S (Fig. 12). However, one noticeable change appears in the upper part where the roll streamlines become inclined from the vertical axis $(z / \delta>5)$. Such inclined streamlines suggest the generation of vertically propagating internal waves. These internal waves are generated because the rolls keep perturbing the stably stratified layer while they propagate horizontally and grow in time. The internal waves have the same horizontal wavenumber $k$, angular frequency $\omega_{r}$, and growth rate $\omega_{i}$ as the rolls. As shown in Fig. 15, the rolls and internal waves are phase locked and coupled together. In the following, we use an analytical model to explore the linkage between the rolls and internal waves and the effect of the mixedlayer height on the internal wave properties.

We consider a region $\left(z>z_{1}\right)$ where the mean wind is weak $(\bar{u} \sim 0)$ and $N^{2}$ is constant. This region will be referred to as region $\Re$. The mean wind shear and $\bar{K}_{m}$ are negligible in region $\Re$. Below region $\Re$, the mean wind shear is relatively stronger and rolls are generated owing to the inflection point instability. In region $\Re$, we can neglect terms in (7)-(10) related to $\bar{u}$ and $\bar{K}_{m}$. Such approximation allows us to derive an analytical solution for internal waves in region $\Re$. Let us consider the internal wave solution in the form of normal mode. For example, the steam function is written as $\psi^{\prime}=\hat{\psi}(z) \exp i(k x-\omega t)$, where $\omega=\omega_{r}+i \omega_{i}$; $k, \omega_{r}$, and $\omega_{i}$ of the internal waves in region $\Re$ are determined by the rolls generated below region $\Re$. By substituting the normal-mode solutions into (7)-(10), we can derive the equation for $\hat{\psi}(z)$ in region $\Re$,

$$
\frac{d^{2} \hat{\psi}}{d z^{2}}+m^{2} \hat{\psi}=0
$$
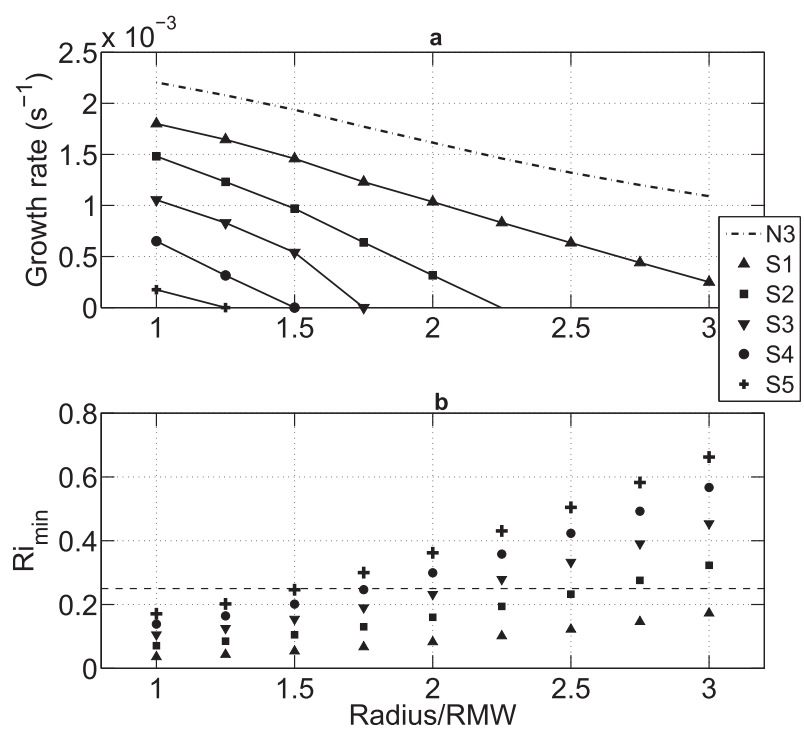

FIG. 11. (a) Growth rate of rolls and (b) the minimum Richardson number as functions of radius in group S. Growth rate of rolls in experiment $\mathrm{N} 3$ (dashed-dotted line) is also shown in (a). The critical Richardson number $(0.25)$ is indicated by the dashed line in (b).

in which

$$
m^{2}=\frac{N^{2}}{c^{2}}-k^{2}
$$
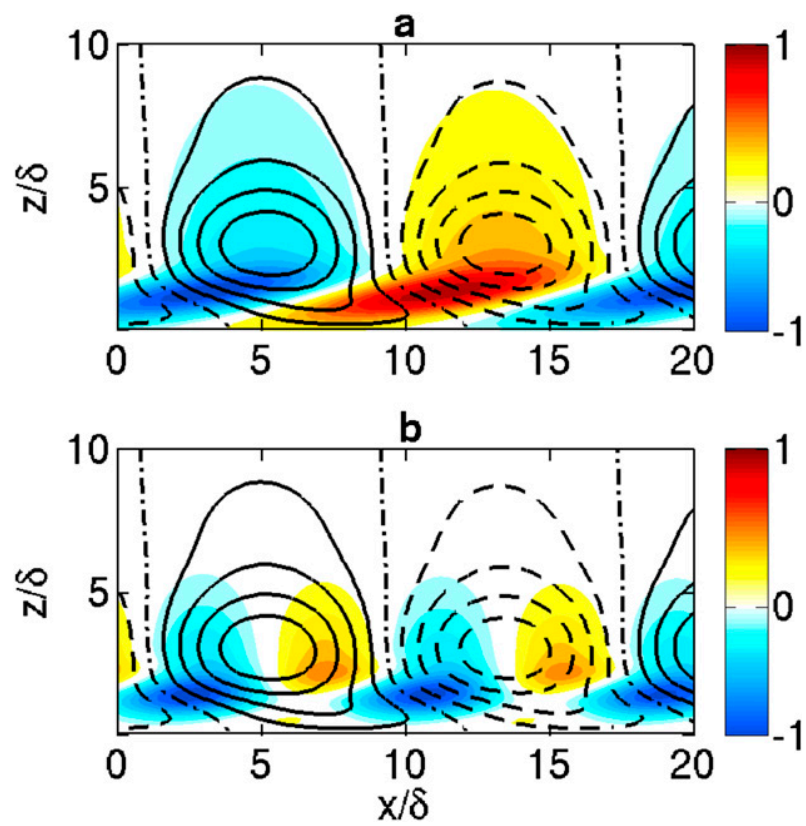

FIG. 12. Typical structure of rolls in group S. The colored backgrounds represent fields for (a) $\theta^{\prime}$ and (b) $w^{\prime} \theta^{\prime}$. These variables are normalized by their maximum values. The $x$ and $z$ axes are nondimensionalized by $\delta$. Contours are streamlines: solid (dashed) contours represent clockwise (counterclockwise) circulations. The rolls shown are at RMW $(\delta=200 \mathrm{~m})$ in experiment S3 with lapse rate of $3 \mathrm{~K} \mathrm{~km}^{-1}$ 


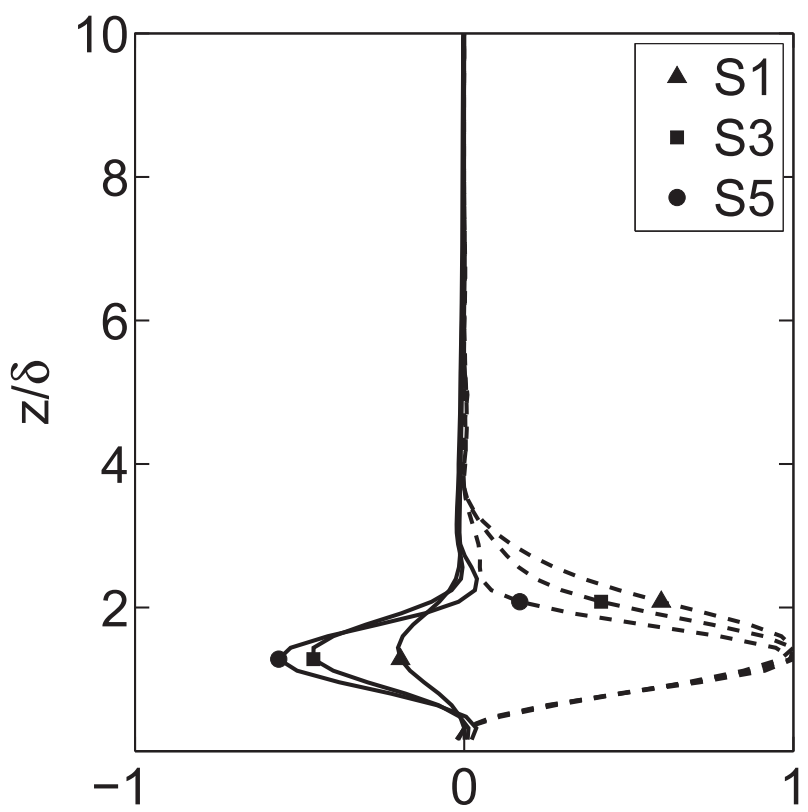

FIG. 13. Vertical distributions of buoyancy work (solid lines) and shear production (dashed lines) in experiments S1, S3, and S5 at RMW. The values shown are nondimensional. Each pair of shear production and buoyancy work profiles is nondimensionalized by dividing the maximum value of the shear production. The vertical axis is nondimensionalized by $\delta(\delta=200 \mathrm{~m})$.

where $c=\omega / k$. Equation (12) has a solution in the form of $\hat{\psi}=\psi_{0} \exp (\mathrm{imz})$, where $\psi_{0}$ is an arbitrary constant. Since $c$ has both the real and imaginary parts, $m$ is a complex number (i.e., $m=m_{r}+i m_{i}$ ). If $N^{2}=0$ (e.g., group N), then according to (13), $m_{r}=0$ and $m_{i}=|k|$, and therefore $\hat{\psi}=\exp (-|k| z)$, which means $\hat{\psi}(z)$ decays exponentially with height. But if $N^{2} \neq 0$ (e.g., groups $\mathrm{M}$ and $\mathrm{S}$ ), $m$ has both nonzero real and imaginary parts, suggesting the internal waves have both vertically propagating and vertically decaying properties.

To characterize the properties of internal waves, we introduce two nondimensional parameters: $\Gamma=m / k$ and $D=\left|m_{r} / m_{i}\right|$. These nondimensonal parameters were first introduced by Sutherland et al. (1994). The real part of $\Gamma$ (i.e., $m_{r} / k$ ), measures the inclination angle $\alpha$ of the internal wave phase lines from the vertical direction, where $\alpha$ is given by $\alpha=\arctan \left(\Gamma_{r}\right)$. Parameter $D$ is the ratio of the vertical $e$-folding length to the vertical wavelength. Following Sutherland et al. (1994), $D$ is referred to as the penetration ratio. Larger $D$ suggests that the internal waves have less vertically decaying property and more vertically propagating property. For example, if $m_{i}$ is zero and $D$ is infinitely large, the internal waves propagate vertically without any decay. After introducing $\Gamma$, (13) becomes

$$
\Gamma^{2}=\frac{N^{2}}{\omega^{2}}-1
$$

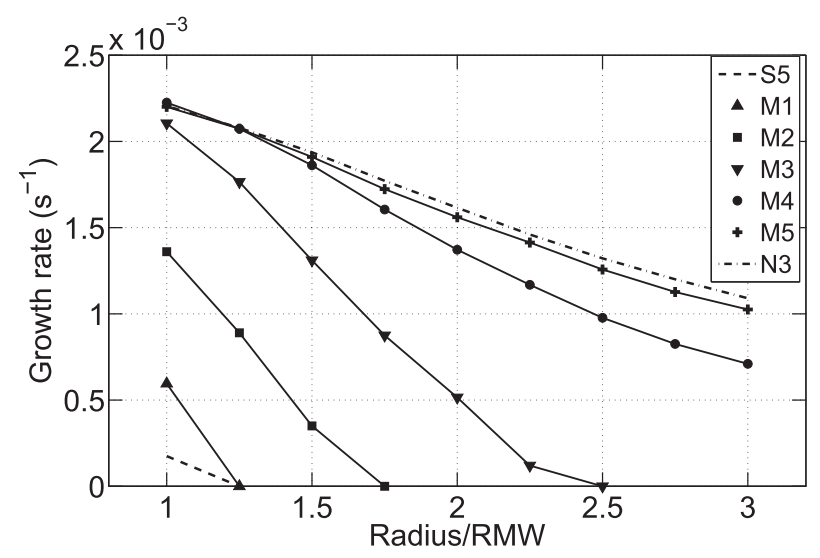

FIG. 14. Growth rate of rolls as function of radius in group M. For comparison, growth rates of rolls in experiments S5 (dashed line) and N3 (dashed-dotted line) are also shown.

Taking square root of (14), we get

$$
\begin{aligned}
\Gamma_{ \pm}= & \pm\left[\sqrt{\frac{C_{1}+\sqrt{C_{1}^{2}+C_{2}^{2}}}{2}}\right. \\
& \left.+i \operatorname{sgn}\left(C_{2}\right) \sqrt{\frac{-C_{1}+\sqrt{C_{1}^{2}+C_{2}^{2}}}{2}}\right]
\end{aligned}
$$

where $C_{1}=N^{2}\left(\omega_{r}^{2}-\omega_{i}^{2}\right) /\left(\omega_{r}^{2}+\omega_{i}^{2}\right)^{2}-1, \quad C_{2}=$ $-2 N^{2} \omega_{r} \omega_{i} /\left(\omega_{r}^{2}+\omega_{i}^{2}\right)^{2}$, and sgn is the sign function. Also, $\omega_{r}$ and $\omega_{i}$ are both positive, and therefore $\operatorname{sgn}\left(C_{2}\right)=-1$. We only allow vertically bounded solutions (i.e., $m_{i}>0$ ).

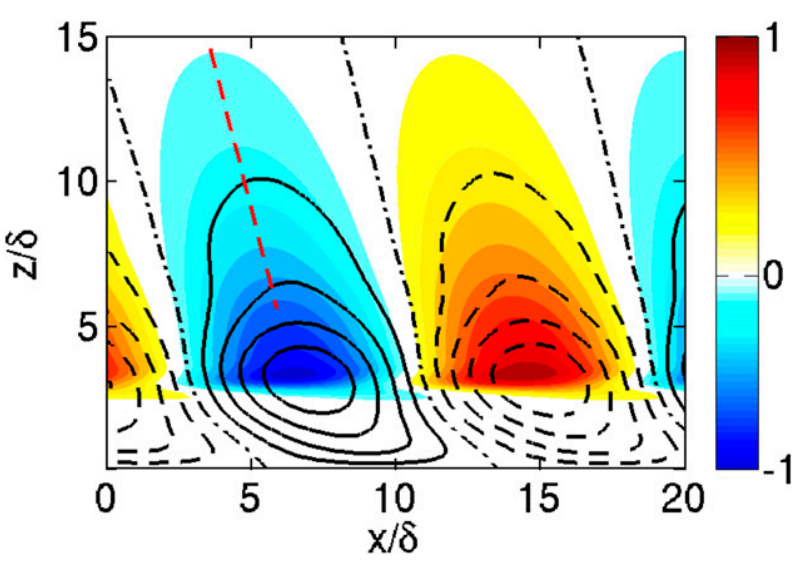

FIG. 15. Typical structure of rolls in group M. The colored background represents $\theta^{\prime}$ normalized by its maximum value. The $x$ and $z$ axes are nondimensionalized by $\delta$. Contours are streamlines: solid (dashed) contours represent clockwise (counterclockwise) circulations. The rolls shown are at RMW $(\delta=200 \mathrm{~m})$ in experiment M5 with $z_{i}=576 \mathrm{~m}$. The angle between the red dashed line and the vertical axis is $\alpha$ calculated based on (16). 
This requires $\Gamma_{i}<0$ because the horizontal wavenumber is always negative (rolls always propagate in the negative $x$ direction). So we only keep $\Gamma_{+}$in (15). Thus we have

$$
\begin{aligned}
& \Gamma_{r}=\sqrt{\frac{C_{1}+\sqrt{C_{1}^{2}+C_{2}^{2}}}{2},} \\
& \Gamma_{i}=-\sqrt{\frac{-C_{1}+\sqrt{C_{1}^{2}+C_{2}^{2}}}{2}}, \text { and } \\
& D=\frac{\Gamma_{r}}{\Gamma_{i}}=\frac{C_{1}+\sqrt{C_{1}^{2}+C_{2}^{2}}}{C_{2}} .
\end{aligned}
$$

The solutions (16) and (18) allow to determine $\alpha$ and $D$ of the internal waves generated by rolls with known $\omega_{r}$ and $\omega_{i}$. To validate the analytical solutions, we compare the inclination angles estimated based on (16) with the inclination angles of the upper-level structures derived by the SRM in group M. The roll growth rate is calculated using the method described in section $3 \mathrm{~b}$. The roll angular frequency is derived from Fourier analysis of the time series of $w^{\prime}$ at a fixed point within the mixed layer. The angular frequency corresponding to the peak of the spectrum is considered to be the angular frequency of the rolls. As seen in Fig. 15, the inclination angles in the analytical and numerical solutions match very well.

Figure 16 shows $\alpha$ and $D$, derived based on (16) and (18), as functions of the mixed-layer height at RMW. As the mixed-layer height increases, the inclination angle of the internal wave phase lines also increases. This implies that the phase lines of internal waves are more inclined from the vertical direction when the mixed layer gets higher. The penetration ratio also increases with the increasing mixed-layer height, suggesting that the internal waves generated by the rolls have less vertically decaying property and more vertically propagating property under nonuniform stratification with a higher mixed layer.

\section{Discussion}

In the three groups of experiments described above, we assume that the angle between the along-roll axis and the azimuthal direction $\varepsilon$ is zero. To explore the effect of this assumption on the roll characteristics, we performed an additional group of experiments to test how sensitive the rolls are to the choice of $\varepsilon$. In these experiments, we used the basic-state HBL wind distribution in experiment $\mathrm{N} 3\left(l_{\infty}=40 \mathrm{~m}\right)$ and assumed that the stratification is neutral. We varied $\varepsilon$ from $-10^{\circ}$ to $15^{\circ}$ with an interval of $5^{\circ}$ (positive $\varepsilon$ means the along-roll axis is to the left of the azimuthal direction). Figure 17 shows the growth rates of rolls as functions of $\varepsilon$ at different locations. The results suggest the angle at which rolls reach the largest growth rate is within $10^{\circ}$ (slightly to the left of the local azimuthal wind direction), which is consistent with Foster (2005). The assumption $\varepsilon=0$ underestimates the roll growth rate by a small factor $(10 \%$ at most). The roll wavelengths change insignificantly from $0^{\circ}$ to $10^{\circ}$ (not shown).

In group N, we vary the basic-state HBL wind distribution by changing $l_{\infty}$. An alternative way to vary the basic-state HBL wind distribution is to change the $V_{g}$ distribution at the upper boundary of the basic-state HBL model. Following KW01, in addition to the storm used in group N (i.e., storm I in KW01), we considered another two storms: a less initially stable storm (storm II in KW01) and a more intense storm (storm III in KW01). Similar to the effect of $l_{\infty}$ in group N, the radial distribution of $V_{g}$ affected the dynamical boundary layer height and the wind shear and, thus, affected the wavelength and growth rate of rolls. We found the overall radial tendencies of the wavelength and growth rate of rolls under storms II and III were qualitatively similar to those under storm I.

According to our analysis, the spatial scale of rolls is proportional to $\delta$, which depends on $I$ and $\bar{K}_{m}$. The rolls obtained in group $\mathrm{N}$ have generally larger wavelengths than those in Foster (2005), primarily because we applied a larger turbulent viscosity to set up the basic-state HBL winds. In fact, by reducing $l_{\infty}$ in group $\mathrm{N}$ (e.g., $l_{\infty}=$ $5 \mathrm{~m}$ ), we can derive rolls with the wavelength similar to those in Foster (2005). The dependency of rolls on the HBL height may explain the difference between the rolls derived by Nolan (2005) and Foster (2005). Rolls derived by Nolan (2005) have wavelengths in the range of $3-5 \mathrm{~km}$, much larger than the rolls derived by Foster (2005). However, this does not necessarily mean the roll formation mechanisms are different in these two studies. The difference in the wavelengths between Nolan (2005) and Foster (2005) likely originates from the different choices of the gradient wind distribution and the turbulent viscosity, both affecting the dynamical HBL height and ultimately the wavelength of rolls. The dependency of roll wavelength on the dynamical HBL height may also give some explanation to the variability of the roll wavelength in the observations. The variability of observed roll wavelength could result from the variability of dynamical height associated with the mean HBL wind distribution.

Previous theoretical studies (Foster 2005; Nolan 2005) primarily focused on the effect of the mean HBL wind structure on the rolls, but the effect of stratification has not been fully investigated. Nolan (2005) used neutral stratification throughout the atmospheric layer that he considered. Foster (2005) included the near-surface 

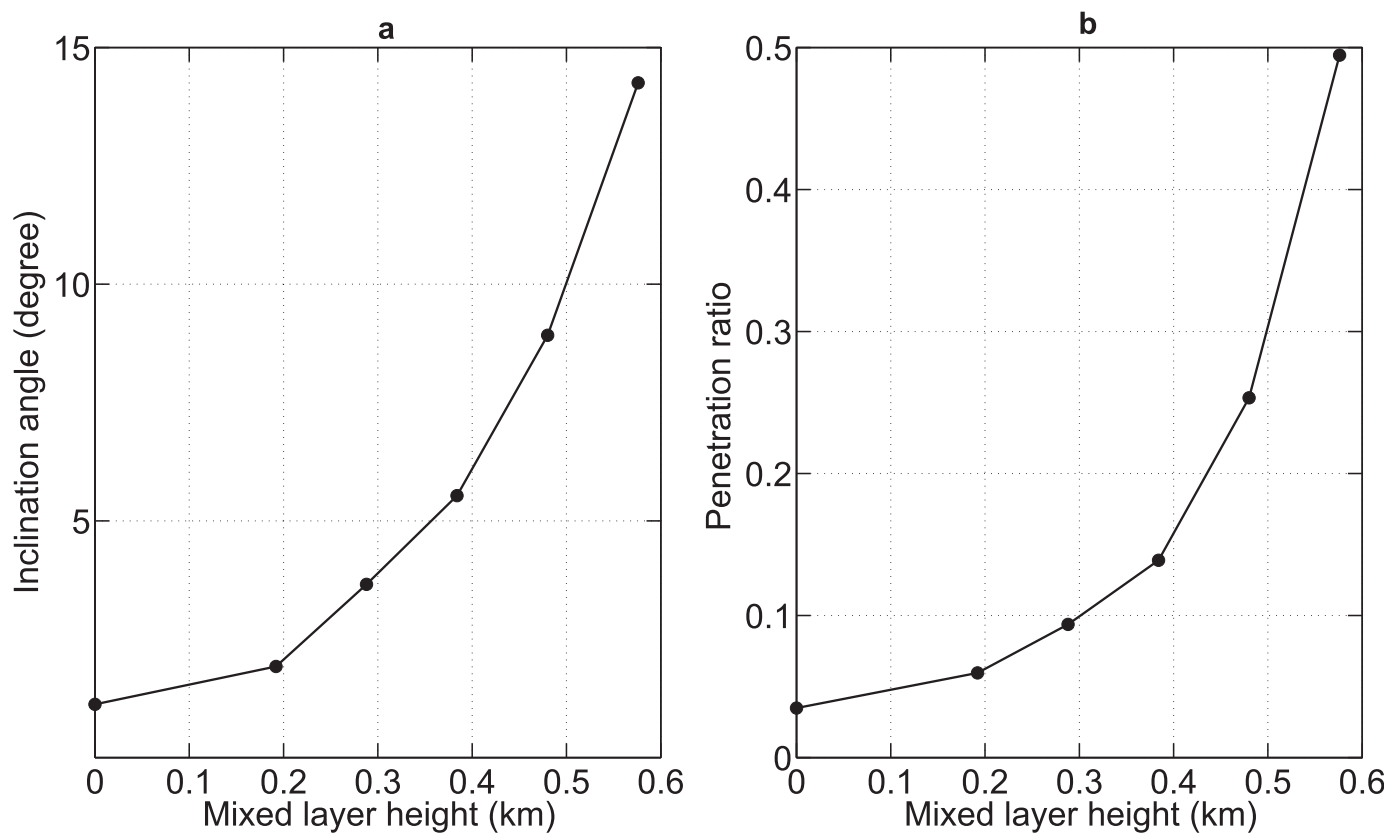

FIG. 16. (a) Inclination angle and (b) the penetration ratio of the internal waves as functions the mixed-layer height at RMW calculated based on (16) and (18), respectively. The roll growth rates and angular frequencies from experiments S5 and M1-M5 are used.

unstably stratified layer but he did not consider the stably stratified layer above the mixed layer. The unstably stratified layer Foster (2005) considered did not appear to affect roll characteristics significantly. This study emphasizes the effects of stratification on rolls. We find that the stable stratification in the HBL may suppress the inflection point instability under some conditions. This may explain why rolls do not always exist in the HBL observations (e.g., Morrison et al. 2005) even though the inflection point in the radial wind profile is always present. Moreover, we find the stably stratified layer provides the environment for rolls to couple with internal waves. The characteristics of rolls and internal waves are affected by the mixed-layer height, suggesting the mixed-layer height is a critical length scale.

Spatial resolutions of the majority of current hurricane models are too coarse to resolve rolls and therefore their effects are not explicitly included. To properly parameterize the effects of rolls, process-oriented studies need to be conducted to study their dynamics, such as their formation and nonlinear evolution. One possible limitation of our numerical approach is the assumption of two-dimensional structure of rolls. Although this assumption can be justified because rolls tend to be elongated along the mean wind direction within the HBL, a high-resolution three-dimensional LES model would be the best tool for studying rolls in hurricanes. However, it is not practical at present because of computer limitations.
Utilization of the SRM imbedded into a HBL model allows us to simplify the problem and dramatically decrease the amount of computation. This numerical approach can serve as a useful tool to study both the thermal and inflection point instabilities in the HBL and the effects of the environmental factors such as the mean wind and stratification. Because the equations describing the HBL

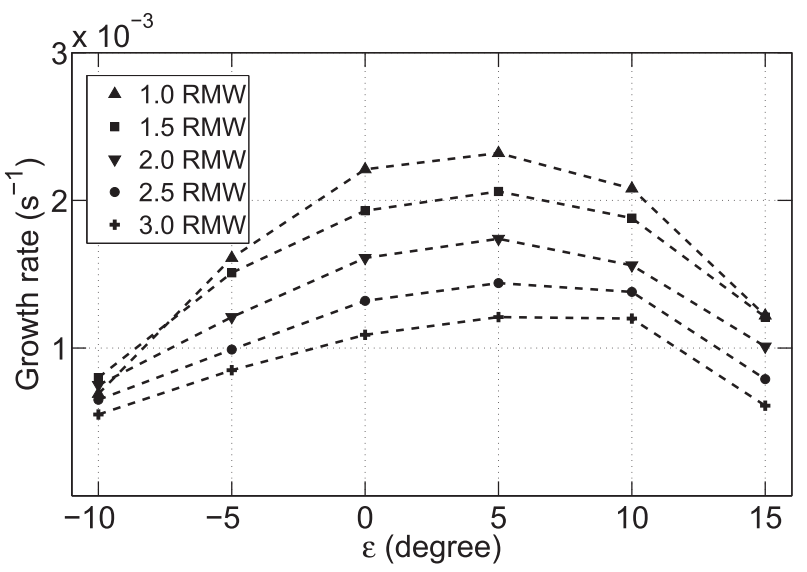

FIG. 17. Growth rates of rolls as functions of $\varepsilon$ at different locations. In these experiments, the basic-state HBL wind distribution is as in experiment $\mathrm{N} 3\left(l_{\infty}=40 \mathrm{~m}\right)$ and no stratification is considered. We define $\varepsilon$ as the angle between the along-roll direction and the azimuthal direction, as shown in Fig. 1. 
mean flow and the SRM are coupled, this approach can be further extended to study the nonlinear phase of rolls, their interactions with the internal waves, as well as their interactions with the mean HBL flow.

\section{Conclusions}

We have investigated the role of the mean wind (large-scale wind) and the stratification on the inflection point instability in the hurricane boundary layer (HBL). The study was performed using a two-dimensional Single-Grid Roll-Resolving Model (SRM) to resolve linearphase rolls under various HBL conditions. The key findings in this study are summarized as follows. Rolls generated by the inflection point instability are characterized by tilted streamlines in the vicinity of the inflection point of the radial wind profile. Kinetic energy budget consideration reveals that the tilted streamlines are critical for the rolls to extract kinetic energy from the mean wind. We have identified two important factors of the mean HBL winds that affect the characteristics of rolls: the dynamical HBL height affects the wavelength of rolls, and the magnitude of the mean wind shear affects the growth rate of rolls. Therefore, under neutrally stratified HBL, the wavelength of rolls increases with the distance from the storm center (outside of the RMW), while their growth rate decreases. The stable stratification in the HBL can suppress the growth of rolls, and rolls can be generated only if the minimum gradient Richardson number is less than 0.25 . The nonuniform stratification with a mixed layer has less suppressing effect on rolls. If the mixed layer is sufficiently high, the stably stratified layer above has minor effect on the growth of rolls.

Rolls generated by the inflection point instability can trigger internal waves in the stably stratified layer, which have the same horizontal wavelength, growth rate, and angular frequency as rolls. These internal waves have both vertically propagating and decaying properties. We derived analytical solutions for internal waves, which relate the properties of the internal waves to the properties of boundary layer rolls. We find as the mixed-layer height increases, the phase lines of the internal waves are more inclined from the vertical direction, and the internal waves have less vertically decaying property and more vertically propagating property.

Since there is a growing interest in parameterizing the roll-induced mixing effect in hurricane models, we think it is necessary to outline the important environmental factors affecting the formation of rolls and their characteristics. We believe that this work improves the current understanding on rolls in the HBL and provides important guidelines for the parameterization of rolls.
Acknowledgments. We acknowledge Ralph Foster for his valuable input throughout this study and Jun Zhang for providing the composite hurricane boundary layer observations. We thank Jun Zhang and one anonymous reviewer for providing the insightful comments that significantly improved this work. This research was funded by the Office of Navy Research through Award N000141210447.

\section{APPENDIX}

\section{Equations for the Mean Flow and Roll Vortices in the Hurricane Boundary Layer}

The governing equations for the dry atmosphere in the hurricane boundary layer under Boussinesq assumption are

$$
\begin{aligned}
\frac{\partial \mathbf{v}}{\partial t}+\mathbf{v} \cdot \nabla \mathbf{v}+f \mathbf{k} \times \mathbf{v} & =-\frac{1}{\rho_{0}} \nabla p+\frac{\theta^{\prime}}{\theta_{0}} \mathbf{g}+K_{m} \nabla^{2} \mathbf{v} \\
\nabla \cdot \mathbf{v} & =0, \quad \text { and } \\
\frac{\partial \theta}{\partial t}+\mathbf{v} \cdot \nabla \theta & =K_{h} \nabla^{2} \theta
\end{aligned}
$$

where $\mathbf{v}$ is the velocity vector and $\theta$ is the potential temperature; $K_{m} \nabla^{2} \mathbf{v}$ and $K_{h} \nabla^{2} \theta$ represent mixing effect of small-scale turbulences in the boundary layer, where $K_{m}$ and $K_{h}$ are turbulent viscosity and diffusivity, respectively, and need to be parameterized. Based on the flow-separation assumption described in section 2, the governing equations for the mean flow (denoted by the overbar) and rolls (denotes by the prime) can be derived from (A1)-(A3). These equations are presented below.

\section{a. Equations for the mean flow}

We assume the large-scale hurricane flow is stationary and axisymmetric. The governing equations for the mean flow in the cylindrical coordinates $(r, \lambda, z)$ are

$$
\begin{gathered}
\frac{\partial \bar{U}}{\partial t}+\bar{U} \frac{\partial \bar{U}}{\partial r}+\bar{W} \frac{\partial \bar{U}}{\partial z}-\frac{\bar{V}^{2}}{r}-f \bar{V} \\
=-\frac{1}{\rho_{0}} \frac{\partial \bar{P}}{\partial r}-\frac{\partial \overline{W^{\prime} U^{\prime}}}{\partial z}+\bar{K}_{m} \frac{\partial^{2} \bar{U}}{\partial z^{2}}, \\
\frac{\partial \bar{V}}{\partial t}+\bar{U} \frac{\partial \bar{V}}{\partial r}+\bar{W} \frac{\partial \bar{V}}{\partial z}+\frac{\bar{U} \bar{V}}{r}+f \bar{U} \\
=-\frac{\partial \overline{W^{\prime} V^{\prime}}}{\partial z}+\bar{K}_{m} \frac{\partial^{2} \bar{V}}{\partial z^{2}}, \\
\frac{\partial \bar{U}}{\partial r}+\frac{\bar{U}}{r}+\frac{\partial \bar{W}}{\partial z}=0, \quad \text { and }
\end{gathered}
$$




$$
\frac{\partial \bar{\theta}}{\partial t}+\bar{U} \frac{\partial \bar{\theta}}{\partial r}+\bar{W} \frac{\partial \bar{\theta}}{\partial z}=-\frac{\partial \overline{W^{\prime} \theta^{\prime}}}{\partial z}+\bar{K}_{h} \frac{\partial^{2} \bar{\theta}}{\partial z^{2}}
$$

where $\bar{U}, \bar{V}$, and $\bar{W}$ are the mean wind components in the radial, azimuthal, and vertical directions, respectively.
Terms like $\overline{W^{\prime} U^{\prime}}$ are the horizontally averaged fluxes induced by rolls.

\section{b. Equations for rolls}

The perturbation equations in the cylindrical coordinates are

$$
\begin{gathered}
\frac{\partial U^{\prime}}{\partial t}+U^{\prime} \frac{\partial U^{\prime}}{\partial r}+\frac{V^{\prime}}{r} \frac{\partial U^{\prime}}{\partial \lambda}+W^{\prime} \frac{\partial U^{\prime}}{\partial z}=-\bar{U} \frac{\partial U^{\prime}}{\partial r}-\frac{\bar{V}}{r} \frac{\partial U^{\prime}}{\partial \lambda}-W^{\prime} \frac{\partial \bar{U}}{\partial z}+\frac{2 \bar{V}}{r} V^{\prime}+f V^{\prime}-\frac{1}{\rho_{0}} \frac{\partial p^{\prime}}{\partial r}+K_{m} \nabla_{r}^{2} U^{\prime}, \\
\frac{\partial V^{\prime}}{\partial t}+U^{\prime} \frac{\partial V^{\prime}}{\partial r}+\frac{V^{\prime}}{r} \frac{\partial V^{\prime}}{\partial \lambda}+W^{\prime} \frac{\partial V^{\prime}}{\partial z}=-\bar{U} \frac{\partial V^{\prime}}{\partial r}-\frac{\bar{V}}{r} \frac{\partial V^{\prime}}{\partial \lambda}-W^{\prime} \frac{\partial \bar{V}}{\partial z}-\frac{\bar{V}}{r} U^{\prime}-\frac{\bar{U}}{r} V^{\prime}-f U^{\prime}-\frac{1}{r \rho_{0}} \frac{\partial p^{\prime}}{\partial \lambda}+K_{m} \nabla_{r}^{2} V^{\prime}, \\
\frac{\partial W^{\prime}}{\partial t}+U^{\prime} \frac{\partial W^{\prime}}{\partial r}+\frac{V^{\prime}}{r} \frac{\partial W^{\prime}}{\partial \lambda}+W^{\prime} \frac{\partial W^{\prime}}{\partial z}=-\bar{U} \frac{\partial W^{\prime}}{\partial r}-\frac{\bar{V}}{r} \frac{\partial W^{\prime}}{\partial \lambda}-\frac{1}{\rho_{0}} \frac{\partial p^{\prime}}{\partial z}+g \frac{\theta^{\prime}}{\theta_{0}}+K_{m} \nabla_{r}^{2} W^{\prime}, \\
\frac{\partial U^{\prime}}{\partial r}+\frac{U^{\prime}}{r}+\frac{1}{r} \frac{\partial V^{\prime}}{\partial \lambda}+\frac{\partial W^{\prime}}{\partial z}=0, \quad \text { and } \\
\frac{\partial \theta^{\prime}}{\partial t}+U^{\prime} \frac{\partial \theta^{\prime}}{\partial r}+\frac{V^{\prime}}{r} \frac{\partial \theta^{\prime}}{\partial \lambda}+W^{\prime} \frac{\partial \theta^{\prime}}{\partial z}=-\bar{U} \frac{\partial \theta^{\prime}}{\partial r}-\frac{\bar{V}}{r} \frac{\partial \theta^{\prime}}{\partial \lambda}-W^{\prime} \frac{\partial \bar{\theta}}{\partial z}+K_{h} \nabla_{r}^{2} \theta^{\prime},
\end{gathered}
$$

where $\nabla_{r}^{2}=\partial^{2} / \partial r^{2}+(1 / r)(\partial / \partial r)+\left(1 / r^{2}\right)\left(\partial^{2} / \partial \lambda^{2}\right)+\partial^{2} / \partial z^{2}$ and $U^{\prime}, V^{\prime}$, and $W^{\prime}$ are the wind perturbations in the radial, azimuthal, and vertical directions, respectively.

Similar to Foster (2005), we use a local Cartesian coordinate system $(x, y, z)$ instead of the cylindrical coordinates at large radius (e.g., radius larger than the radius of maximum wind). We assume rolls are aligned along the $y$ axis and the along-roll variations are negligible. The governing equations for rolls in the local Cartesian coordinates at radius $r=R$ are

$$
\begin{aligned}
\frac{\partial u^{\prime}}{\partial t}+u^{\prime} \frac{\partial u^{\prime}}{\partial x}+w^{\prime} \frac{\partial u^{\prime}}{\partial z}= & -\bar{u} \frac{\partial u^{\prime}}{\partial x}-w^{\prime} \frac{\partial \bar{u}}{\partial z}-\frac{1}{\rho_{0}} \frac{\partial p^{\prime}}{\partial x} \\
& +\frac{1}{R}\left[(\bar{v} \sin \varepsilon) u^{\prime}+(2 \bar{v} \cos \varepsilon\right. \\
& \left.+\bar{u} \sin \varepsilon) v^{\prime}\right]+f v^{\prime}+K_{m} \nabla^{2} u^{\prime},
\end{aligned}
$$

(A8)

$$
\begin{aligned}
\frac{\partial v^{\prime}}{\partial t}+u^{\prime} \frac{\partial v^{\prime}}{\partial x}+w^{\prime} \frac{\partial v^{\prime}}{\partial z}= & -\bar{u} \frac{\partial v^{\prime}}{\partial x}-w^{\prime} \frac{\partial \bar{v}}{\partial z} \\
& -\frac{1}{R}\left[(2 \bar{u} \sin \varepsilon+\bar{v} \cos \varepsilon) u^{\prime}\right. \\
& \left.+(\bar{u} \cos \varepsilon) v^{\prime}\right]-f u^{\prime}+K_{m} \nabla^{2} v^{\prime}
\end{aligned}
$$

$\frac{\partial w^{\prime}}{\partial t}+u^{\prime} \frac{\partial w^{\prime}}{\partial x}+w^{\prime} \frac{\partial w^{\prime}}{\partial z}=-\bar{u} \frac{\partial w^{\prime}}{\partial x}-\frac{1}{\rho_{0}} \frac{\partial p^{\prime}}{\partial z}+g \frac{\theta^{\prime}}{\theta_{0}}+K_{m} \nabla^{2} w^{\prime}$,

$$
\frac{\partial u^{\prime}}{\partial x}+\frac{\partial w^{\prime}}{\partial z}=0, \quad \text { and }
$$

$\frac{\partial \theta^{\prime}}{\partial t}+u^{\prime} \frac{\partial \theta^{\prime}}{\partial x}+w^{\prime} \frac{\partial \theta^{\prime}}{\partial z}=-\bar{u} \frac{\partial \theta^{\prime}}{\partial x}-w^{\prime} \frac{\partial \bar{\theta}}{\partial z}+K_{h} \nabla^{2} \theta^{\prime}$,

where $\nabla^{2}=\partial^{2} / \partial x^{2}+\partial^{2} / \partial z^{2},\left(u^{\prime}, v^{\prime}, w^{\prime}\right)$ are the roll velocities in the local Cartesian coordinates and $\bar{u}$ and $\bar{v}$ are the mean winds in the $x$ and $y$ directions, respectively.

Next we introduce the along-roll vorticity $\eta^{\prime}$ and the streamfunction $\psi^{\prime}$ to simplify the roll equations. The alongroll vorticity $\left(\eta^{\prime}=\partial w^{\prime} / \partial x-\partial u^{\prime} / \partial z\right)$ is related to $\psi^{\prime}\left(u^{\prime}=\right.$ $-\partial \psi^{\prime} / \partial z$ and $\left.w^{\prime}=\partial \psi^{\prime} / \partial x\right)$ by the Poisson's equation:

$$
\eta^{\prime}=\frac{\partial^{2} \psi^{\prime}}{\partial x^{2}}+\frac{\partial \psi^{\prime}}{\partial z^{2}}
$$

The equation for $\eta^{\prime}$ can be derived from (A13) and (A15), which is

$$
\begin{aligned}
\frac{\partial \eta^{\prime}}{\partial t} & +u^{\prime} \frac{\partial \eta^{\prime}}{\partial x}+w^{\prime} \frac{\partial \eta^{\prime}}{\partial z}=-\bar{u} \frac{\partial \eta^{\prime}}{\partial x}+w^{\prime} \frac{\partial^{2} \bar{u}}{\partial z^{2}}+\frac{g}{\theta_{0}} \frac{\partial \theta^{\prime}}{\partial x} \\
& -\frac{1}{R} \frac{\partial\left[(\bar{v} \sin \varepsilon) u^{\prime}+(2 \bar{v} \cos \varepsilon+\bar{u} \sin \varepsilon) v^{\prime}\right]}{\partial z} \\
& -f \frac{\partial v^{\prime}}{\partial z}+K_{m} \nabla^{2} \eta^{\prime}
\end{aligned}
$$

The new set of equations for rolls is given by (A9) and (A12)-(A14).

The numerical methods applied for solving the meanflow and roll equations are described in Ginis et al. (2004). 


\section{REFERENCES}

Blackadar, A. K., 1962: The vertical distribution of wind and turbulent exchange in a neutral atmosphere. J. Geophys. Res., 67, 3095-3102, doi:10.1029/JZ067i008p03095.

Brown, R. A., 1970: A secondary flow model of the planetary boundary layer. J. Atmos. Sci., 27, 742-757, doi:10.1175/ 1520-0469(1970)027<0742:ASFMFT>2.0.CO;2.

- 1972: On the inflection point instability of a stratified Ekman boundary layer. J. Atmos. Sci., 29, 850-859, doi:10.1175/ 1520-0469(1972)029<0850:OTIPIO > 2.0.CO;2.

Donelan, M. A., B. K. Haus, N. Reul, W. J. Plant, M. Stiassnie, H. C. Graber, O. B. Brown, and E. S. Saltzman, 2004: On the limiting aerodynamic roughness of the ocean in very strong winds. Geophys. Res. Lett., 31, L18306, doi:10.1029/2004GL019460.

Ellis, R., and S. Businger, 2010: Helical circulation in the typhoon boundary layer. J. Geophys. Res., 115, D06205, doi:10.1029/ 2009JD011819.

Faller, A. J., 1965: Large eddies in the atmospheric boundary layer and their possible role in the formation of cloud rows. J. Atmos. Sci., 22, 176-184, doi:10.1175/1520-0469(1965)022<0176 LEITAB $>2.0$. CO 2 .

Foster, R. C., 2005: Why rolls are prevalent in the hurricane boundary layer. J. Atmos. Sci., 62, 2647-2661, doi:10.1175/ JAS3475.1.

— , 2009: Boundary-layer similarity under an axisymmetric, gradient wind vortex. Bound.-Layer Meteor., 131, 321-344, doi:10.1007/s10546-009-9379-1.

Ginis, I., A. P. Khain, and E. Morozovsky, 2004: Effects of large eddies on the structure of the marine boundary layer under strong wind conditions. J. Atmos. Sci., 61, 3049-3064, doi:10.1175/JAS-3342.1

Holland, G. J., 1980: An analytic model of the wind and pressure profiles in hurricanes. Mon. Wea. Rev., 108, 1212-1218, doi:10.1175/1520-0493(1980)108<1212:AAMOTW>2.0.CO;2.

Katsaros, K. B., P. W. Vachon, P. G. Black, P. P. Dodge, and E. W Uhlhorn, 2000: Wind fields from SAR: Could they improve our understanding of storm dynamics? Johns Hopkins APL Tech. Dig., 21, 86-93.

Kepert, J. D., 2001: The dynamics of boundary layer jets within the tropical cyclone core. Part I: Linear theory. J. Atmos. Sci., 58, 2469 2484, doi:10.1175/1520-0469(2001)058<2469:TDOBLJ>2.0.CO;2. , 2012: Choosing a boundary layer parameterization for tropical cyclone modeling. Mon. Wea. Rev., 140, 1427-1445, doi:10.1175/MWR-D-11-00217.1.

_, and Y. Wang, 2001: The dynamics of boundary layer jets within the tropical cyclone core. Part II: Nonlinear enhancement. J. Atmos. Sci., 58, 2485-2501, doi:10.1175/ 1520-0469(2001)058<2485:TDOBLJ > 2.0.CO;2.

Lilly, D., 1966: On the instability of Ekman boundary flow. J. Atmos. Sci., 23, 481-494, doi:10.1175/1520-0469(1966)023<0481: OTIOEB $>2.0 . \mathrm{CO} ; 2$.

Lorsolo, S., J. L. Schroeder, P. Dodge, and F. Marks, 2008: An observational study of hurricane boundary layer small-scale coherent structures. Mon. Wea. Rev., 136, 2871-2893, doi:10.1175/2008MWR2273.1.

Miles, J. W., 1961: On the stability of heterogenous shear flows. J. Fluid Mech., 10, 496-508, doi:10.1017/S0022112061000305.

Moon, I.-J., I. Ginis, T. Hara, and B. Thomas, 2007: A physicsbased parameterization of air-sea momentum flux at high wind speeds and its impact on hurricane intensity predictions. Mon. Wea. Rev., 135, 2869-2878, doi:10.1175/MWR3432.1.

Morrison, I., S. Businger, F. Marks, P. Dodge, and J. Businger, 2005: An observational case for the prevalence of roll vortices in the hurricane boundary layer. J. Atmos. Sci., 62, 2662-2673, doi:10.1175/JAS3508.1

Nakanishi, M., and H. Niino, 2012: Large-eddy simulation of roll vortices in a hurricane boundary layer. J. Atmos. Sci., 69, 35583575, doi:10.1175/JAS-D-11-0237.1.

Nolan, D. S., 2005: Instabilities in hurricane-like boundary layers. Dyn. Atmos. Oceans, 40,209-236, doi:10.1016/j.dynatmoce.2005.03.002.

Powell, P., J. Vickery, and T. A. Reinhold, 2003: Reduced drag coefficient for high wind speeds in tropical cyclone. Nature, 422, 279-283, doi:10.1038/nature01481.

Sutherland, B. R., C. P. Caulfield, and W. R. Peltier, 1994: Internal gravity wave generation and hydrodynamic instability. J. Atmos. Sci., 51, 3261-3280, doi:10.1175/1520-0469(1994)051<3261: IGWGAH $>2.0 . \mathrm{CO} ; 2$.

Wurman, J., and J. Winslow, 1998: Intense sub-kilometer boundary layer rolls in Hurricane Fran. Science, 280, 555-557, doi:10.1126/ science.280.5363.555.

Zhang, J. A., and W. M. Drennan, 2012: An observational study of vertical eddy diffusivity in the hurricane boundary layer. J. Atmos. Sci., 69, 3223-3236, doi:10.1175/JAS-D-11-0348.1.

, K. B. Katsaros, P. G. Black, S. Lehner, J. R. French, and W. M. Drennan, 2008: Effects of roll vortices on turbulent fluxes in the hurricane boundary layer. Bound.-Layer Meteor., 128, 173-189, doi:10.1007/s10546-008-9281-2.

—, R. F. Rogers, D. S. Nolan, and F. D. Marks Jr., 2011: On the characteristic height scales of the hurricane boundary layer. Mon. Wea. Rev., 139, 2523-2535, doi:10.1175/MWR-D-10-05017.1.

Zhu, P., 2008: Simulation and parameterization of the turbulent transport in the hurricane boundary layer by large eddies. J. Geophys. Res., 113, D17104, doi:10.1029/2007JD009643. 